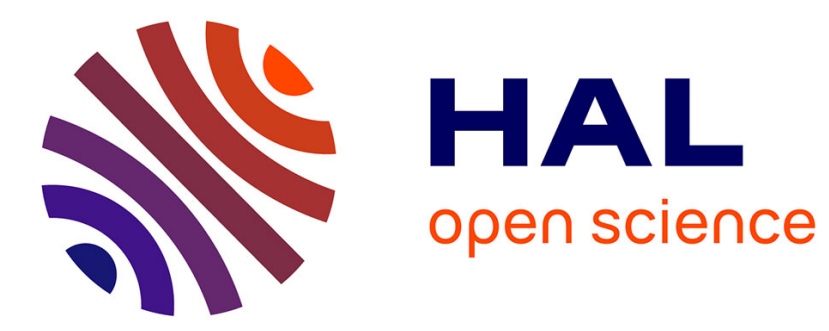

\title{
A Neumann-Neumann Domain Decomposition Algorithm for Solving Plate and Shell Problems
}

\author{
Patrick Le Tallec, Jan Mandel, Marina Vidrascu
}

\section{To cite this version:}

Patrick Le Tallec, Jan Mandel, Marina Vidrascu. A Neumann-Neumann Domain Decomposition Algorithm for Solving Plate and Shell Problems. [Research Report] RR-2635, INRIA. 1995. inria00077196

\section{HAL Id: inria-00077196 \\ https://hal.inria.fr/inria-00077196}

Submitted on 29 May 2006

HAL is a multi-disciplinary open access archive for the deposit and dissemination of scientific research documents, whether they are published or not. The documents may come from teaching and research institutions in France or abroad, or from public or private research centers.
L'archive ouverte pluridisciplinaire HAL, est destinée au dépôt et à la diffusion de documents scientifiques de niveau recherche, publiés ou non, émanant des établissements d'enseignement et de recherche français ou étrangers, des laboratoires publics ou privés. 
INSTITUT NATIONAL DE RECHERCHE EN INFORMATIQUE ET EN AUTOMATIQUE

\section{A Neumann-Neumann Domain Decomposition Algorithm for Solving Plate and Shell Problems}

Patrick Le Tallec , Jan Mandel. , Marina Vidrascu

$\mathbf{N}^{\circ} \mathbf{2 6 3 5}$

August 1995

PROGRAMME 6

\section{apport \\ de recherche}





\title{
A Neumann-Neumann Domain Decomposition Algorithm for Solving Plate and Shell Problems*
}

\author{
Patrick Le Tallec ${ }^{* *}$, Jan Mandel ${ }^{* * *}$., Marina Vidrascu**** \\ Programme 6 - Calcul scientifique, modélisation et logiciel numérique \\ Projet MENUSIN
}

Rapport de recherche $\mathrm{n}^{\circ} 2635$ - August 1995 - 41 pages

\begin{abstract}
We present a new Neumann-Neumann type preconditioner of large scale linear systems arising from plate and shell problems. The advantage of the new method is a smaller coarse space than earlier method of the authors, which improves parallel scalability. A new abstract framework for Neumann-Neumann preconditioners is used to prove optimal convergence properties of the method. The convergence estimates are independent of the number of subdomains, coefficient jumps between subdomains, and depend only polylogarithmically on the number of elements per subdomain. We formulate and prove an approximate parametric variational principle for Reissner-Mindlin elements as the plate thickness approaches zero, which makes the results applicable to a large class of non-locking elements in everyday engineering use. The theoretical results are confirmed by computational experiments on model problems as well as examples from real world engineering practice.
\end{abstract}

Key-words: Iterative methods, preconditioning, domain decomposition, substructuring, coarse problem, balancing, plates, shells, parametric variational principles.

(Résumé : tsvp)

\footnotetext{
* This work was partially supported by INRIA, NSF grants ASC-9217394, ASC-9121431, INT9310529, and ONR grant N-00014-95-1-0663.

** INRIA, Domaine de Voluceau, 78153 Le Chesnay Cedex, France.

Email: Patrick.LeTallec@inria.fr

*** Center for Computational Mathematics, University of Colorado at Denver, Denver, CO 80217 3364, USA. Electronic mail: jmandel@colorado.edu

**** INRIA, Domaine de Voluceau, 78153 Le Chesnay Cedex, France.

Email: Marina.Vidrascu@inria.fr
}

Unité de recherche INRIA Rocquencourt

Domaine de Voluceau, Rocquencourt, BP 105, 78153 LE CHESNAY Cedex (France)

Téléphone : (33 1) 39635511 - Télécopie : (33 1) 39635330 


\section{Un Algorithme de Décomposition de Domaines de type Neumann-Neumann pour les Problèmes de Plaques et Coques}

Résumé : Cet article introduit un nouveau préconditionneur de type NeumannNeumann pour la solution numérique sur machines parallèles de grands problèmes de plaques ou de coques approchés par éléments finis. Cette méthode utilise un espace grossier réduit par rapport à une première version introduite par les auteurs. La convergence optimale de la méthode est démontrée grâce à l'introduction d'un nouveau cadre abstrait qui permet d'analyser de manière systématique les préconditionneurs de type Neumann-Neumann. L'estimation de convergence est indépendante du nombre de sous-domaines, des sauts de coefficients élastiques entre sous-domaines, et ne dépend que logarithmiquement du nombre d'éléments finis par sous-domaine. Nous introduisons aussi un principe variationnel paramétrique approché de type Reissner-Mindlin, ce qui permet d'appliquer nos résultats à un très grand nombre d'éléments finis de plaques utilisés en pratique. Les résultats théoriques sont confirmés par diverses expériences numériques effectuées à la fois sur de petits problèmes modèles et sur des cas industriels significatifs.

Mots-clé : Méthodes itératives, gradient conjugué, préconditionnement, décomposition de domaines, sous-structuration, problèmes sur grille grossière, plaques, coques, principes variationnels paramétriques. 
1. Introduction. The application of domain decomposition methods to the numerical solution of plate or shell problems faces two difficulties: the very poor conditioning of the associated algebraic operator, and the strong continuity requirements needed at each interface. On the other hand, the partition of plate and shell structures into connected substructures is quite a natural operation; direct substructuring has been used for a long time in Engineering. Such direct techniques are successful but are restricted to partitions into small numbers of subdomains and are hard to implement on parallel machines.

The purpose of this paper is to introduce an iterative substructuring technique for thin plates and shells structures which has good parallel and convergence properties and which can handle partitions into a large number of subdomains. The present method is a generalization of the technique we have first described in [25], which uses a Neumann-Neumann preconditioner with a coarse grid solver. The local poor conditioning of the algebraic operator and the interface continuity requirements are handled by the local Neumann operator. A coarse grid solver is added in order to facilitate exchange of information between the subdomains and thus assure that the convergence does not deteriorate with the number of subdomains [27]. The main idea in [25] was to adapt the method of [27] by enlarging the coarse space to enforce continuity of the solution at subdomain corners throughout the iteration. This gave good convergence for plate problems. Here we present an improved method, which reduces significantly the size of the coarse problem while preserving the good convergence properties of the method from [25]. We present a new abstract framework for Neumann-Neumann methods and prove the usual optimal domain decomposition estimate: the condition number grows only weakly with the number of elements per subdomain, and it is bounded independently of the number of subdomains, and of coefficient jumps.

The Neumann-Neumann preconditioner with a coarse space was introduced in [27] under the name Balancing Domain Decomposition and further studied in $[12,28$, 29]. Our abstract framework and analysis are related to the representation of another Neumann-Neumann method with a coarse space as an abstract Schwarz method in [16]. For earlier work on the Neumann-Neumann and similar preconditioners without a coarse space, see $[1,13,14,21,32]$. A domain decomposition method in a sense dual to Neumann-Neumann is obtained by enforcing intersubdomain continuity by Lagrange multipliers $[19,35,17]$. That method, known as FETI, gives rise to a natural coarse problem $[18,30]$. A version of FETI with convergence properties for plates similar to the present method, is based on enforcing the continuity of solution at subdomain corners, also by enlarging the coarse space [31].

$\mathrm{RR} \mathrm{n}^{\circ} 2635$ 
In Section 2, we review the Kirchhoff-Love thin plate model discretized by conforming reduced HCT triangles [11] or approximated by DKT triangles [2]. We show that the local stiffness matrices of these elements are spectrally equivalent, and prove also spectral equivalence, uniformly as the plate thickness approaches zero, for a class of non-locking Reissner-Mindlin elements used in everyday engineering practice. This approximate parametric variational principle will allow us to restrict convergence proofs to the HCT element. The iterative substructuring algorithm is then introduced in Section 3 within a new general framework, which can describe and analyse Neumann-Neumann preconditioners in all generality. In Section 4, we prove several technical lemmas and apply them to the abstract framework to obtain a bound on the condition number. The paper is concluded by a discussion on implementation issues and a description of the numerical tests, which have been run to validate the algorithm for both plate and shell problems in Section 5 .

\section{Finite Elements for Plates.}

2.1. Conforming Approximation of the Kirchhoff-Love model. Let us consider a plate occupying a domain $\Omega$ in $\mathbb{R}^{2}$, which is clamped on the part $\partial \Omega_{c}$ of its boundary and simply supported along $\partial \Omega_{s}-\partial \Omega_{c}$ (Figure 1). Then, a KirchhoffLove model characterizes the vertical displacement $u$ of the plate as the solution of the variational problem

$$
a(u, v)=L(v), \forall v \in \mathbb{H}(\Omega), u \in \mathbb{H}(\Omega),
$$

under the notation

$$
\begin{aligned}
a(u, v) & =\int_{\Omega} \varepsilon(\vec{\theta}(u)): K: \varepsilon(\vec{\theta}(v)), \\
L(v) & =\int_{\Omega} f v+\int_{\partial \Omega-\partial \Omega_{c}} m_{g} \partial_{n} v+\int_{\partial \Omega-\partial \Omega_{s}} g v, \\
\mathbb{H}(\Omega) & =\left\{v \in H^{2}(\Omega), v=0 \quad \text { on } \quad \partial \Omega_{s}, \partial_{n} v=0 \quad \text { on } \quad \partial \Omega_{c}\right\} .
\end{aligned}
$$

Above, $f$ denotes the density of vertical forces, $m_{g}$ the density of flexion moments applied on the part $\partial \Omega-\partial \Omega_{c}$ of the boundary where the plate is free to rotate, $g$ is the density of boundary vertical loading and $\mathbb{H}(\Omega)$ denotes the space of kinematically admissible displacement fields.

Moreover $\varepsilon(\vec{\theta})=\frac{1}{2}\left(\nabla \vec{\theta}+\nabla^{T} \vec{\theta}\right)$ is the curvature tensor, $\vec{\theta}(u)=\nabla u$ represents the inplane rotation of the plate, $K$ the plate flexural stiffness. For a simple isotropic 


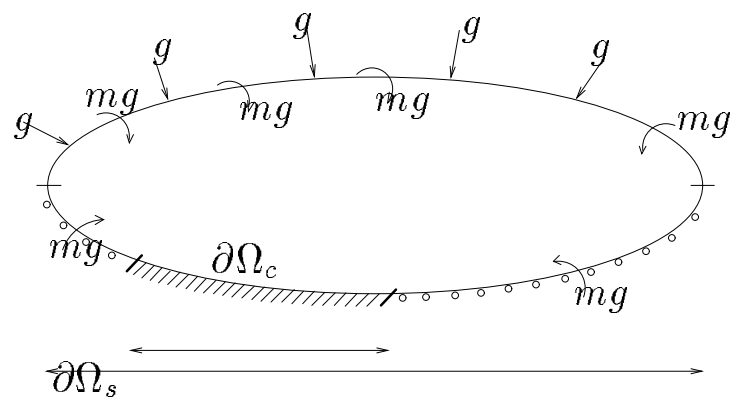

FIG. 1. Plate configuration. The plate is clamped on $\partial \Omega_{c}$ and simply supported on $\partial \Omega_{s}-\partial \Omega_{c}$.

plate of thickness $t$, made of an homogeneous elastic material with Young modulus $E$ and Poisson coefficient $\nu$, this flexural stiffness is given by

$$
\varepsilon(\vec{\theta}(u)): K: \varepsilon(\vec{\theta}(v))=\frac{E t^{3}}{12\left(1-\nu^{2}\right)}\left((1-\nu) \nabla^{2} u: \nabla^{2} v+\nu \Delta u \Delta v\right) .
$$

In the general case, $K$ has a more complex writing but is always symmetric, elliptic, continuous :

$$
\begin{aligned}
\varepsilon(\vec{\theta}): K: \varepsilon(\vec{\theta}) & \geq c_{\alpha} t^{3}|\varepsilon(\vec{\theta})|^{2}, \\
\varepsilon(\vec{\theta}): K: \varepsilon\left(\vec{\theta}^{\prime}\right) & \leq c_{a} t^{3}|\varepsilon(\vec{\theta})|\left|\varepsilon\left(\vec{\theta}^{\prime}\right)\right| .
\end{aligned}
$$

The HCT finite element approximation of the above plate problem is then simply obtained by partitioning $\Omega$ into a regular triangulation $\Omega=\cup_{T} T$ (Fig. 2) and by replacing $\mathbb{H}(\Omega)$ in $(1)$ by the finite element space

$$
\begin{gathered}
\mathbb{H}_{h}(\Omega)=\left\{v \in \mathbb{H}(\Omega),\left.v\right|_{T} \in C^{1}(T),\left.v\right|_{T_{i}} \in P_{3}\left(T_{i}\right), \text { for any subtriangle } T_{i} \text { of } T,\right. \\
\left.\left.\partial_{n} v\right|_{a_{i} a_{j}} \in P_{1}\left(a_{i} a_{j}\right) \text { for any side } a_{i} a_{j} \text { of the triangle } T\right\} .
\end{gathered}
$$

Elements $v$ of this space are characterized by their values $v\left(a_{i}\right)$ and the values of their derivatives $D v\left(a_{i}\right)$ at each vertex $a_{i}$ of the triangulation (Fig. 3). The position of the internal vertex $a_{T}$ is not arbitrary but is determined in order to guarantee the unisolvence of the element :

For any order $\alpha=0, x_{1}, x_{2}$ of the derivative, for any node $i$, there exists then a unique shape function $\varphi_{\alpha i}$ such that

$$
\left.\varphi_{\alpha i}\right|_{T} \in C^{1}(T)
$$




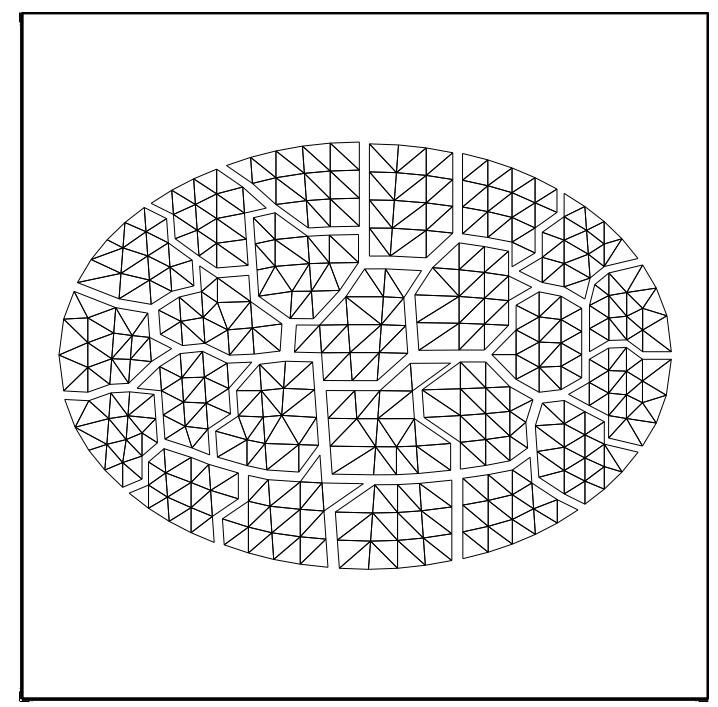

FIG. 2. Triangulation and decomposition of the plate in nonoverlapping subdomains

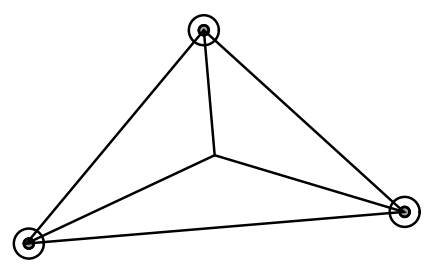

FIG. 3. Reduced HCT triangle. Displacements are characterized by their values and the values of their derivatives at each vertex. 


$$
\begin{aligned}
& \left.\varphi_{\alpha i}\right|_{T_{j}} \in P_{3}\left(T_{j}\right), \quad \forall \text { subtriangle } T_{j} \text { of } T, \\
& \left.\partial_{n} \varphi_{\alpha i}\right|_{a_{j} a_{k}} \in P_{1}\left(a_{j} a_{k}\right), \\
& \partial_{\beta} \varphi_{\alpha i}\left(a_{j}\right)=\delta_{i j} \delta_{\alpha \beta} .
\end{aligned}
$$

We refer to $[5,11]$ for more details on this element.

2.2. DKT Triangle. In a Discrete Kirchhoff Triangle, the vertical displacement $u$ of the plate and its inplane rotation $\vec{\theta}$ are treated as two separate variables. On each triangle $T$ of the triangulation, the displacement $u(M)$ is a third order polynomial of the barycentric coordinates $\lambda_{i}$ of $M$

$$
u(M)=\sum_{i}\left(a_{i} \lambda_{i}+b_{i} \lambda_{i} \lambda_{i+1}+c_{i} \lambda_{i}^{2} \lambda_{i+1}\right),
$$

the rotation $\vec{\theta}$ is a reduced second order polynomial

$$
\vec{\theta}=\sum_{i}\left(\vec{\theta}\left(a_{i}\right) \lambda_{i}+4 \alpha_{i} \lambda_{i} \lambda_{i+1} \overrightarrow{a_{i} a_{i+1}}\right)
$$

and $u$ and $\vec{\theta}$ satisfy the tangential Kirchhoff hypothesis

$$
\frac{\partial u}{\partial s}=\vec{\theta} \cdot \frac{\overrightarrow{a_{i} a_{j}}}{\left|a_{i} a_{j}\right|}
$$

along each side $a_{i} a_{j}$ of the triangle. By writing (6) at each vertex $a_{i}$, we first have

$$
\vec{\theta}\left(a_{i}\right)=\nabla u\left(a_{i}\right) .
$$

By integrating (6) along the side $a_{i} a_{j}$, we also get

$$
u\left(a_{i+1}\right)-u\left(a_{i}\right)=\frac{1}{2}\left(\vec{\theta}\left(a_{i}\right)+\vec{\theta}\left(a_{i+1}\right)\right) \cdot \overrightarrow{a_{i} a_{i+1}}+\frac{2}{3} \alpha_{i}\left|a_{i} a_{i+1}\right|^{2} .
$$

Therefore, the discrete Kirchhoff hypothesis (6) gives the local rotation $\left.\vec{\theta}\right|_{T}$ as a function of $u$ by

$$
\left.\vec{\theta}\right|_{T}=\vec{\theta}_{d}(u):=\sum_{i}\left(\nabla u\left(a_{i}\right) \lambda_{i}+6 \nabla_{i i+1}^{2}(u) \frac{\overrightarrow{a_{i} a_{i+1}}}{\left|a_{i} a_{i+1}\right|} \lambda_{i} \lambda_{i+1}\right),
$$

where

$$
\nabla_{i j}^{2}(u)=\frac{1}{\left|a_{i} a_{j}\right|}\left[u\left(a_{j}\right)-u\left(a_{i}\right)-\frac{1}{2}\left(\nabla u\left(a_{i}\right)+\nabla u\left(a_{j}\right)\right) \cdot \overrightarrow{a_{i} a_{j}}\right]
$$


This approximation leads to the following definition of our finite element space

$$
\begin{aligned}
\mathbb{H}_{d}(\Omega)= & \left\{v \in C^{o}(\Omega),\left.v\right|_{T} \text { satisfies }(4), \vec{\theta}_{d}(v) \in C^{o}(\Omega),\right. \\
& \left.v=0 \quad \text { on } \partial \Omega_{s}, \vec{\theta}_{d}(v)=0 \quad \text { on } \partial \Omega_{c}\right\} .
\end{aligned}
$$

Elements $v$ of this space are again characterized by their values $v\left(a_{i}\right)$ and the values of their derivatives $D v\left(a_{i}\right)$ at each vertex $a_{i}$ of the triangulation. The corresponding discrete variational formulation of the plate problem (1) becomes

$$
\int_{\Omega} \varepsilon\left(\vec{\theta}_{d}(u)\right): K: \varepsilon\left(\vec{\theta}_{d}(v)\right)=L(v), \forall v \in \mathbb{H}_{d}(\Omega), u \in \mathbb{H}_{d}(\Omega) .
$$

2.3. Spectral equivalence. The results of this section will allow us to reduce all considerations to the HCT element. First we show that the local stiffness matrices of the reduced HCT triangle and the DKT triangle are spectrally equivalent to a quadratic form based on finite differences of the values of the degrees of freedom.

THEOREM 2.1. Let $T=a_{1} a_{2} a_{3}$ be a given finite element of a shape regular triangulation of $\Omega$. Let $U=\left(\partial_{\alpha} u\left(a_{i}\right)\right),|\alpha| \leq 1$ be the nodal degrees of freedom of T. Let $I_{H C T}(U)=\partial_{\alpha} u\left(a_{i}\right) \varphi_{\alpha i}$ be the local HCT interpolate of $u$ and $Q_{i j}(U)$ be the quadratic form

$$
Q_{i j}(U)=\left|\nabla u\left(a_{i}\right)-\nabla u\left(a_{j}\right)\right|^{2}+\left|\nabla_{i j}^{2} u\right|^{2}, \quad 1 \leq i \neq j \leq 3 .
$$

The form $Q$ and the local stiffness matrix $A_{T}$ of both the reduced HCT and the DKT triangle satisfy then the equivalence property

$$
\begin{aligned}
c_{o}\left|I_{H C T} U\right|_{2,2, T}^{2} & \leq \frac{1}{t^{3}} U^{T} A_{T} U \leq c_{1}\left|I_{H C T} U\right|_{2,2, T}^{2}, \\
c_{2}\left|I_{H C T} U\right|_{2,2, T}^{2} & \leq \sum_{i} Q_{i i+1}(U) \leq c_{3}\left|I_{H C T} U\right|_{2,2, T}^{2}
\end{aligned}
$$

Above, the constant $c_{i}$ are independent of the element and of the plate thickness but $c_{o}$ and $c_{1}$ do depend on the material constants $c_{\alpha}$ and $c_{a}$.

Proof. By construction, the local stiffness matrices are given by

$$
U^{T} A_{T} U=\int_{T} \varepsilon(\vec{\theta}): K: \varepsilon(\vec{\theta})
$$

with $\varepsilon(\vec{\theta})=\varepsilon\left(\vec{\theta}\left(I_{H C T} U\right)\right)=\nabla^{2} I_{H C T} U$ for the HCT triangle and

$$
\varepsilon(\vec{\theta})=\varepsilon\left(\vec{\theta}_{d}(U)\right)=\sum \nabla u\left(a_{i}\right) \varepsilon\left(\lambda_{i}\right)+6 \nabla_{i i+1}^{2}(u) \frac{\overrightarrow{a_{i} a_{i+1}}}{\left|a_{i} a_{i+1}\right|} \varepsilon\left(\lambda_{i} \lambda_{i+1}\right)
$$


for the DKT triangle. From the ellipticity conditions (2)-(3), we can therefore deduce

$$
c_{\alpha}|\varepsilon(\vec{\theta})|_{0,2, T}^{2} \leq \frac{1}{t^{3}} U^{T} A_{T} U \leq c_{a}|\varepsilon(\vec{\theta})|_{0,2, T}^{2} .
$$

For the reduced HCT triangle, this is exactly (10) with $c_{o}=c_{\alpha}$ and $c_{1}=c_{a}$.

To study the DKT triangle, we first map $T$ to a fixed affine equivalent triangle $\hat{T}$ by the mapping

$$
\begin{aligned}
& \hat{x}:=B x+x, \\
& \hat{U}:=\left(u\left(a_{i}\right), \nabla u\left(a_{i}\right) \cdot \frac{\partial x}{\partial \hat{x}}\right)=\left(u\left(a_{i}\right), \nabla u\left(a_{i}\right) \cdot B^{-1}\right)=\left(\hat{\partial}_{\alpha} \hat{u}\left(\hat{a}_{i}\right)\right)_{i}, \\
& \hat{I}_{H C T} \hat{U}(\hat{x}):=I_{H C T} U(x(\hat{x})):=\hat{\partial}_{\alpha} \hat{u}\left(\hat{a}_{i}\right) \hat{\varphi}_{i \alpha}\left(\hat{a}_{T}, e_{T}, \hat{x}\right) \text {, } \\
& \hat{\nabla}_{i j}^{2} \hat{U}:=\frac{1}{\hat{a}_{i} \hat{a}_{j}}\left[\hat{u}\left(a_{i}\right)-\hat{u}\left(a_{j}\right)-\frac{1}{2}\left(\hat{\nabla} \hat{u}\left(\hat{a}_{i}\right)+\hat{\nabla} \hat{u}\left(\hat{a}_{j}\right)\right) \cdot \hat{a}_{i} \hat{a}_{j}\right], \\
& \hat{\theta}_{d}(\hat{U}):=\sum_{i}\left(\hat{\nabla} \hat{u}\left(\hat{a}_{i}\right) \lambda_{i}+6 \hat{\nabla}_{i i+1}^{2} \hat{U} \frac{\hat{a}_{i} \hat{a}_{i+1}}{\left|\hat{a}_{i} \hat{a}_{i+1}\right|} \lambda_{i} \lambda_{i+1}\right) \\
& \hat{\varepsilon}(\hat{\theta}):=\frac{1}{2}\left(\hat{\nabla} \hat{\theta}+\hat{\nabla}^{T} \hat{\theta}\right), \\
& \hat{Q}_{i j}(\hat{U}):=\left|\hat{\nabla} \hat{u}\left(\hat{a}_{i}\right)-\hat{\nabla} \hat{u}\left(\hat{a}_{j}\right)\right|^{2}+\left|\hat{\nabla}_{i j}^{2} \hat{U}\right|^{2} .
\end{aligned}
$$

In the reference triangle $\hat{T}$, the nodal shape function $\hat{\varphi}_{\alpha i}\left(\hat{a}_{T}, e_{T}, \hat{x}\right)$ are piecewise third order polynomials in $\hat{x}$ whose coefficients depend continuously on the position $\hat{a}_{T}$ of the internal vertex of the HCT triangle and on the eccentricity $e_{T}$ of $T[11,5]$. By construction, if $h_{T}$ and $\rho_{T}$ denote the diameter and the internal radius of the triangle $T$, the above mappings satisfy

$$
\begin{aligned}
\rho_{T}\left|\nabla u\left(a_{i}\right)\right| & \leq\left|\hat{\nabla} \hat{u}\left(\hat{a}_{i}\right)\right|=\left|\nabla u\left(a_{i}\right) \cdot B^{-1}\right| \leq h_{T}\left|\nabla u\left(a_{i}\right)\right|, \\
\rho_{T}\left|\nabla_{i j}^{2} U\right| & \leq\left|\hat{\nabla}_{i j}^{2} \hat{U}\right|=\frac{\left|a_{i} a_{j}\right|}{\left|\hat{a}_{i} \hat{a}_{j}\right|}\left|\nabla_{i j}^{2} U\right| \leq h_{T}\left|\nabla_{i j}^{2} U\right|, \\
\rho_{T}^{2}\left|I_{H C T} U\right|_{2,2, T}^{2} & \leq\left|\hat{I}_{H C T} \hat{U}\right|_{2,2, \hat{T}}^{2}=\operatorname{det} B\left|\nabla^{2} I_{H C T} U \cdot B^{-2}\right|_{0,2, T}^{2} \leq h_{T}^{2}\left|I_{H C T} U\right|_{2,2, T}^{2},
\end{aligned}
$$

which implies

$$
\begin{aligned}
\rho_{T}^{2} Q_{i j}(U) & \leq \hat{Q}_{i j}(\hat{U}) \leq h_{T}^{2} Q_{i j}(U), \\
\rho_{T}^{2}\left|\varepsilon\left(\theta_{d}\right)\right|_{0,2, T}^{2} & \leq\left|\hat{\varepsilon}\left(\hat{\theta}_{d}\right)\right|_{0,2, \hat{T}}^{2} \leq h_{T}^{2}\left|\varepsilon\left(\theta_{d}\right)\right|_{0,2, T}^{2} .
\end{aligned}
$$

On the other hand,

$$
\left|\hat{I}_{H C T}(\hat{U})\right|_{2,2, \hat{T}}^{2},\left|\hat{\varepsilon}\left(\hat{\theta}_{d}\right)\right|_{0,2, \hat{T}}^{2} \text { and } \sum_{i} \hat{Q}_{i i+1}(\hat{U})
$$

RR $\mathrm{n}^{\circ} 2635$ 
are three quadratic forms defined on $\mathbb{R}^{9}$, operating on the variable $\hat{U}=\left(\hat{\partial}_{\alpha} \hat{u}\left(\hat{a}_{i}\right)\right)$. These quadratic forms are independent of $T$ with the exception of $\hat{I}_{H C T}(\hat{U})$ which depends continuously on $\hat{a}_{T}$ and $e_{T}$. Let us compute their kernels.

The last form $\sum_{i} \hat{Q}_{i+1}(\hat{U})$ cancels if and only if $\hat{\nabla} \hat{u}\left(\hat{a}_{i}\right)=\hat{\nabla} \hat{u}\left(\hat{a}_{j}\right)$, and $\hat{u}\left(\hat{a}_{j}\right)=\hat{u}\left(\hat{a}_{i}\right)+\hat{\nabla} \hat{u}\left(\hat{a}_{i}\right) \cdot \hat{a}_{i} \hat{a}_{j}$ for all $i \neq j$. In other words, the kernel of this last form is

$$
\begin{aligned}
\hat{P}_{1} & =\left\{\hat{U}, \hat{\nabla} \hat{u}\left(\hat{a}_{i}\right)=\hat{\theta}_{o}, \hat{u}\left(\hat{a}_{i}\right)=\hat{u}_{o}+\hat{\theta}_{o} . \hat{a}_{i}\right\} \\
& =\left\{\hat{U}, I_{H C T}(\hat{U})(\hat{x})=\hat{u}_{o}+\hat{\theta}_{o} . \hat{x}\right\}
\end{aligned}
$$

The first form $\left|I_{H C T}(\hat{U})\right|_{2,2, \hat{T}}^{2}$ cancels if and only if $I_{H C T}(\hat{U})$ is a linear function, which means that its kernel is again $\hat{P}_{1}$. Finally, $|\hat{\varepsilon}(\hat{\theta})|_{0,2, \hat{T}}^{2}$ cancels if and only if $\hat{\theta}$ is a pure rotation

$$
\hat{\theta}(\hat{x})=\hat{\theta}_{o}+\omega e_{z} \times \hat{x}
$$

By construction of $\hat{\theta}$, this is equivalent to

$$
\hat{\nabla}_{i j}^{2} \hat{U}=0, \quad \forall i \neq j, \quad \hat{\nabla} \hat{u}\left(a_{i}\right)=\hat{\theta}_{o}+\omega e_{z} \times \hat{a}_{i} .
$$

By summation, this implies

$$
0=\sum_{i}\left|\hat{a}_{i} \hat{a}_{i+1}\right| \hat{\nabla}_{i i+1}^{2} \hat{U}=\sum_{i} \hat{\nabla} \hat{u}\left(\hat{a}_{i}\right) \cdot \hat{a}_{i-1} \hat{a}_{i+1}=\sum_{i} \omega \operatorname{det}\left(e_{z}, \hat{a}_{i}, \hat{a}_{i-1} \hat{a}_{i+1}\right)
$$

which can occur only if $\omega=0$. Therefore the kernel of $|\hat{\varepsilon}(\hat{\theta})|_{0,2, \hat{T}}^{2}$ is again $\hat{P}_{1}$.

Because all three quadratic forms have the same kernels on $\mathbb{R}^{9}$ the limits

$$
\begin{aligned}
& c_{o}\left(\hat{a}_{T}, \hat{e}_{T}\right)=\inf _{\hat{U} \notin \hat{P}_{1}} \frac{|\hat{\varepsilon}(\hat{\theta})|_{0,2, \hat{T}}^{2}}{\left|\hat{I}_{H C T} \hat{U}\right|_{2,2, \hat{T}}^{2}}, \\
& c_{1}\left(\hat{a}_{T}, \hat{e}_{T}\right)=\sup _{\hat{U} \notin \hat{P}_{1}} \frac{|\hat{\varepsilon}(\hat{\theta})|_{0,2, \hat{T}}^{2}}{\left|\hat{I}_{H C T} \hat{U}\right|_{2,2, \hat{T}}^{2}}, \\
& c_{2}\left(\hat{a}_{T}, \hat{e}_{T}\right)=\inf _{\hat{U} \notin \hat{P}_{1}} \frac{\left|\sum Q_{i i+1}(\hat{U})\right|}{\left|\hat{I}_{H C T} \hat{U}\right|_{2,2, \hat{T}}^{2}}, \\
& c_{3}\left(\hat{a}_{T}, \hat{e}_{T}\right)=\sup _{\hat{U} \notin \hat{P}_{1}} \frac{\left|\sum Q_{i i+1}(\hat{U})\right|}{\left|\hat{I}_{H C T} \hat{U}\right|_{2,2, \hat{T}}^{2}},
\end{aligned}
$$


are strictly positive continuous functions of $\hat{a}_{T}$ and $e_{T}$. Since these numbers belong to compact subsets of $\hat{T}$ and $\mathbb{R}$, these functions $c_{i}\left(\hat{a}_{T}, e_{T}\right)$ are uniformly bounded above and below in $\mathbb{R}^{+}$by positive constant $\hat{c}_{i}$.

Combining (16)-(19) with (14)-(15) then yields

$$
\begin{aligned}
\frac{\rho_{T}^{2}}{h_{T}^{2}} \hat{c}_{o}\left|I_{H C T} U\right|_{2,2, T}^{2} \leq \quad|\varepsilon(\theta)|_{0,2, T}^{2} & \leq \frac{h_{T}^{2}}{\rho_{T}^{2}} \hat{c}_{1}\left|I_{H C T} U\right|_{2,2, T}^{2}, \\
\frac{\rho_{T}^{2}}{h_{T}^{2}} \hat{c}_{2}\left|I_{H C T} U\right|_{2,2, T}^{2} \leq \sum_{i} Q_{i i+1}(U) & \leq \frac{h_{T}^{2}}{\rho_{T}^{2}} \hat{c}_{3}\left|I_{H C T} U\right|_{2,2, T}^{2},
\end{aligned}
$$

which is the desired result.

The next theorem allows us to treat a class of non-locking Reissner-Mindlin triangular and quadrilateral elements commonly used in engineering practice [3, 34]. We show that for such elements, the element energy is spectrally equivalent to the same finite difference quadratic form as in the preceding theorem. The essential property of such elements is that as the thickness goes to zero, the stiffness matrix approaches a matrix for a thin plate model rather than to degenerate and cause locking. Such property has been called a parametric variatonal principle; for example, a Timoshenko beam element becomes in the thin limit a cubic spline for the biharmic equation [20]. We have only an approximate version of this property here, which is enough for our purposes.

On a general mesh consisting of triangles or quadrilaterals, we also define a $C^{1}$ interpolation by dividing each quadrilateral into triangles, on which we define the HCT shape functions. The resulting interpolation will be denoted again $I_{H C T}$.

Theorem 2.2 (Approximate parametric Variational principle). Let $T=a_{1} a_{2} a_{3} a_{4}$ or $T=a_{1} a_{2} a_{3}$ be a given finite element in a shape regular "triangulation" of $\Omega$, with the vertex degrees of freedom $\partial_{\alpha} u\left(a_{i}\right)$, local stiffness matrix $A_{T}$, and such that the finite element space on $T$ contains the space $P_{1}$ of all linear functions. Let the element energy satisfy the equivalence property

$$
c \frac{1}{t^{3}} U^{T} A_{T} U \leq \mathcal{E}_{T}(U) \leq C \frac{1}{t^{3}} U^{T} A_{T} U
$$

where

$$
\mathcal{E}_{T}(U)=h^{2}\left(\int_{T}|\nabla \vec{\theta}|^{2}+\frac{1}{t^{2}+h^{2}} \int_{T}|\vec{\theta}-\nabla u|^{2}\right)
$$

$\vec{\theta}$ is the $P_{1}$ or $Q_{1}$ interpolation of the rotational degrees of freedom $\partial_{\alpha} u\left(a_{i}\right),|\alpha|=1$, and $u$ is the $P_{1}$ or $Q_{1}$ (i.e., linear or bilinear for a quadrilateral) interpolation of 
the displacement degrees of freedom $u\left(a_{i}\right)$. Then, for some constants $c_{0}, c_{1}, c_{2}, c_{3}$,

$$
\begin{aligned}
c_{o}\left|I_{H C T} U\right|_{2,2, T}^{2} & \leq \frac{1}{t^{3}} U^{T} A_{T} U \leq c_{1}\left|I_{H C T} U\right|_{2,2, T}^{2}, \\
c_{2} \frac{1}{t^{3}} U^{T} A_{T} U & \leq \sum_{i} Q_{i i+1}(U) \leq c_{3} \frac{1}{t^{3}} U^{T} A_{T} U,
\end{aligned}
$$

for all $0 \leq t \leq h$, where

$$
Q_{i j}(U)=\left|\nabla u\left(a_{i}\right)-\nabla u\left(a_{j}\right)\right|^{2}+\left|\nabla_{i j}^{2} u\right|^{2}, \quad 1 \leq i \neq j \leq 3 \text { or } 4 .
$$

Proof. For $0 \leq t \leq h$, we have

$$
\frac{1}{2}\left(h^{2} \int_{T}|\nabla \vec{\theta}|^{2}+\int_{T}|\vec{\theta}-\nabla u|^{2}\right) \leq \mathcal{E}(U) \leq h^{2} \int_{T}|\nabla \vec{\theta}|^{2}+\int_{T}|\vec{\theta}-\nabla u|^{2} .
$$

Next we map $T$ on a reference element $\hat{T}$, and let

$$
\hat{\mathcal{E}}(\hat{U})=\int_{T}|\hat{\nabla} \overrightarrow{\hat{\theta}}|^{2}+\int_{T}|\overrightarrow{\hat{\theta}}-\nabla \hat{u}|^{2} .
$$

The degrees of freedom scale as

$$
u\left(a_{i}\right)=\hat{u}\left(\hat{a}_{i}\right), \quad \overrightarrow{\hat{\theta}} \approx h \vec{\theta},
$$

and $\hat{Q}_{i j}$ is as in (13). Since $P_{1}$ is contained in the element space, from the definition of $\mathcal{E}, \hat{\mathcal{E}}(U)=0$ if and only if $\hat{u} \in P_{1}$ and $\overrightarrow{\hat{\theta}}=\nabla \hat{u}$. But this is if and only if $\sum_{i} Q_{i i+1}(\hat{U})=0$. Thus, by finite dimension, for some $c>0, C$,

$$
c \hat{\mathcal{E}}(\hat{U}) \leq \sum_{i} \hat{Q}_{i i+1}(\hat{U}) \leq C \hat{\mathcal{E}}(\hat{U})
$$

and the proof is concluded by mapping from $\hat{T}$ back to $T$. $\square$

REMARK 2.3. The essential properties of the element used in the proof of Theorem 2.2 are (i) invariance of the energy functional to dilation, with proper scaling of the energy and of the rotational degrees of freedom, and (ii) the zero energy modes being exactly $P_{1}$ functions. 
2.4. Thin shells extension. The standard Koiter's theory of linearly elastic thin shells characterises the three-dimensional displacement $\underline{u}$ of the shell middle surface $\omega$ as the minimiser of its mechanical energy :

$$
\min _{\underline{u} \in \underline{\mathbb{H}}(\Omega)}\left\{\frac{1}{2} \int_{\omega} t^{3} \gamma(\underline{u}): K: \gamma(\underline{u})+t \varepsilon(\underline{u}): A: \varepsilon(\underline{u})-L(u)\right\} .
$$

For shells $\omega$ which are the image of an open domain $\omega_{o}$ of $\mathbb{R}^{2}$ by the map $\underline{\varphi}$, and with unit normal vector $\underline{n}$, the space $\underline{\mathbb{H}}(\Omega)$ of kinematically admissible displacement fields, the curvature change tensor $\gamma(\underline{u})$ and the membrane strain tensor $\varepsilon(\underline{u})$ are of the form [6] :

$$
\begin{gathered}
\underline{\mathbb{H}}(\Omega)=\left\{\underline{u} \in\left(H_{0}^{1}\left(\omega_{0}\right)\right)^{3}, \nabla^{2} \underline{u} \cdot \underline{n} \in\left(L^{2}(\omega)\right)^{4}\right\} \\
\varepsilon(\underline{u})=\frac{1}{2}\left(\nabla \underline{u}^{t} \cdot \nabla \underline{\varphi}+\nabla \underline{\varphi^{t}} \cdot \nabla \underline{u}\right) \\
\gamma(\underline{u})=-\left(\underline{n} \cdot \nabla^{2} \underline{u}+(\nabla \underline{n} \cdot \underline{u}) \cdot \nabla^{2} \underline{\varphi}\right) .
\end{gathered}
$$

These definitions imply that the elastic energy of the shell

$$
\frac{1}{2} \int_{\omega} t^{3} \gamma(\underline{u}): K: \gamma(\underline{u})+t \varepsilon(\underline{u}): A: \varepsilon(\underline{u})
$$

is no longer equivalent to the $H^{2}$ norm of the displacement but to a combination of the $H^{2}$ norm of the normal displacement $\underline{u} \cdot \underline{n}$ and of a $H^{1}$ norm of $\underline{u}$,

$$
\|\underline{u}\|^{2}=\|\underline{u}\|_{1,2, \omega}^{2}+\left\|\underline{n} \cdot \nabla^{2} \underline{u}\right\|_{0,2, \omega}^{2}
$$

the constant of equivalence being now dependent on the thickness $[4,6]$.

Formally and numerically, all the tools to be introduced in this paper will be extended to this thin shell case or even to thick shells. What is needed is to update the energy form and to replace the normal displacement $u$ of the plate theory by the vector displacement $\underline{u}: \omega_{0} \rightarrow \mathbb{R}^{3}$ and the two dimensional rotation $\vec{\theta}=\nabla u$ by a three dimensional vector $\underline{\theta}$ defined at each finite element node. The tangential component $\underline{\theta}_{t}$ of $\underline{\theta}$ still represent the rotation of the middle surface normal vector $\left(\underline{\theta}_{t}=\nabla \underline{u} . \underline{n}\right.$ for thin shells satisfying the Kirchhoff assumption) and its normal component $\theta_{n}$ is associated to an inplane rotation of the shell middle surface $[3,9,10]$.

The mathematical theory could also be adapted to this case but the predicted speed of convergence of our algorithm will no longer be thickness independent. This is confirmed by numerical experience, cf., Tab. 5 and 4 . 


\section{The Neumann-Neumann substructuring technique.}

3.1. The interface problem. Let us partition our original plate $\Omega$ into nonoverlapping subdomains $\Omega_{i}, i=1, \ldots, N$ (Fig. 2). Denote the interfaces

$$
\Gamma_{i}=\partial \Omega_{i} \backslash \partial \Omega, \quad \Gamma=\cup_{i} \Gamma_{i} .
$$

In what follows, the trace on a given interface $\Gamma_{i}$ is defined by

$$
\left.\operatorname{Tr} u\right|_{\Gamma_{i}}=\left(\left.u\right|_{\Gamma_{i}},\left.\vec{\theta}(u)\right|_{\Gamma_{i}}\right) .
$$

We wish to reduce the solution of our original plate problem (1) to the iterative solution of smaller subproblems set on the different subdomains $\Omega_{i}$. For this purpose, we first introduce our local spaces of restrictions

$$
\begin{aligned}
\mathbb{H}\left(\Omega_{i}\right) & =\left\{v_{i}=\left.v\right|_{\Omega_{i}}, v \in \mathbb{H}(\Omega)\right\}, \\
\stackrel{\circ}{H}\left(\Omega_{i}\right) & =\left\{v_{i} \in \mathbb{H}(\Omega), \operatorname{Tr} v_{i}=0 \text { on } \overline{\Omega \backslash \Omega_{i}}\right\}, \\
& =\text { space of functions of } \mathbb{H}\left(\Omega_{i}\right) \text { with zero trace on } \Gamma_{i} .
\end{aligned}
$$

On $\mathbb{H}\left(\Omega_{i}\right)$, we define the local bilinear form

$$
a_{i}(u, v)=\int_{\Omega_{i}} \varepsilon(\vec{\theta}(u)): K: \varepsilon(\vec{\theta}(v)) .
$$

With this new notation, our original problem takes the variational form

$$
u \in \mathbb{H}(\Omega): \quad \sum_{i=1}^{N}\left(a_{i}(u, v)-L_{i}(v)\right)=0, \quad \forall v \in \mathbb{H}(\Omega) .
$$

In operator form, if $A_{i}$ is the operator associated to the bilinear form (20) and if $R_{i}$ denotes the restriction operator from $\mathbb{H}(\Omega)$ into $\mathbb{H}\left(\Omega_{i}\right)$, this problem is

$$
\sum_{i} R_{i}^{T} A_{i} R_{i} U=\sum_{i} R_{i}^{T} L_{i}
$$

To reduce this global problem to an interface problem, we decompose the degrees of freedom $U_{i}$ of $u_{i}=R_{i} u$ into internal degrees of freedom $\stackrel{\circ}{U}_{i}$ and interface degrees of freedom $\bar{U}_{i}$ :

$$
U_{i}=\left[\begin{array}{c}
\stackrel{\circ}{U}_{i} \\
\bar{U}_{i}
\end{array}\right]
$$


With this decomposition, the operator representations of $a_{i}(.,$.$) and L_{i}($.$) on \mathbb{H}\left(\Omega_{i}\right)$ take the form

$$
A_{i}=\left[\begin{array}{cc}
\stackrel{\circ}{A}_{i} & B_{i} \\
B_{i}^{T} & \bar{A}_{i}
\end{array}\right], \quad L_{i}=\left[\begin{array}{c}
\stackrel{\circ}{L}_{i} \\
\bar{L}_{i}
\end{array}\right]
$$

If we suppose for the moment that $\bar{U}_{i}$ is known, the solution of (22) with test functions $v \in \stackrel{\circ}{H}\left(\Omega_{i}\right)$ yields

$$
\stackrel{\circ}{U}_{i}=\stackrel{\circ}{A}_{i}^{-1}\left(\stackrel{\circ}{L}_{i}-B_{i} \bar{U}_{i}\right) .
$$

To determine the trace $\bar{U}_{i}$, we next introduce the global trace space $\mathrm{V}=$ $\left.\operatorname{Tr} \mathbb{H}(\Omega)\right|_{\Gamma}$, the local trace spaces

$$
\mathrm{V}_{i}=\left\{\bar{v}_{i}=\left.\operatorname{Tr} v_{i}\right|_{\Gamma_{i}}: v_{i} \in \mathbb{H}\left(\Omega_{i}\right)\right\}=\left\{\bar{v}_{i}=\left.\operatorname{Tr} v\right|_{\Gamma_{i}}: v \in \mathbb{H}(\Omega)\right\},
$$

and the discrete $a_{i}$-harmonic extension $\operatorname{Tr}_{i}^{-1}: \mathrm{V}_{i} \rightarrow \mathbb{H}\left(\Omega_{i}\right)$ defined by

$(24) a_{i}\left(\operatorname{Tr}_{i}^{-1} \bar{u}_{i}, v_{i}\right)=0, \quad \forall v_{i} \in \stackrel{\circ}{H}\left(\Omega_{i}\right),\left.\quad \operatorname{Tr}\left(\operatorname{Tr}_{i}^{-1} \bar{u}_{i}\right)\right|_{\Gamma_{i}}=\bar{u}_{i}, \quad \operatorname{Tr}_{i}^{-1} \bar{u}_{i} \in \mathbb{H}\left(\Omega_{i}\right)$.

In matrix form, we have simply

$$
\operatorname{Tr}_{i}^{-1}=\left(\begin{array}{c}
-\stackrel{\circ}{i}_{i}^{-1} B_{i} \\
I
\end{array}\right)
$$

We then define the local Schur complement operator $S_{i}: \mathrm{V}_{i} \rightarrow \mathrm{V}_{i}^{\prime}$ by

$$
\left\langle S_{i} \bar{u}_{i}, \bar{v}_{i}\right\rangle=a_{i}\left(\operatorname{Tr}_{i}^{-1} \bar{u}_{i}, \operatorname{Tr}_{i}^{-1} \bar{v}_{i}\right), \quad \forall \bar{u}_{i}, \bar{v}_{i} \in \mathrm{V}_{i} .
$$

Because of the ellipticity conditions $(2,3)$, the problem (24) is well-posed and $S_{i}$ is a well-defined self adjoint elliptic continuous operator. In matrix form, we have from (23)

$$
S_{i} \bar{U}_{i}=\left(\bar{A}_{i}-B_{i}^{T} \AA_{i}^{-1} B_{i}\right) \bar{U}_{i} .
$$

Finally, we introduce the interface restriction operator $\bar{R}_{i}: \mathrm{V} \rightarrow \mathrm{V}_{i}$ by

$$
\bar{R}_{i} \bar{u}=\left.\bar{u}\right|_{\Gamma_{i}}, \forall \bar{u} \in \mathrm{V},
$$

the global Schur complement operator

$$
S=\sum_{i=1}^{N} \bar{R}_{i}^{T} S_{i} \bar{R}_{i},
$$

$\operatorname{RR} n^{\circ} 2635$ 
and the interface right hand side

$$
F=\sum_{i=1}^{N} \bar{R}_{i}^{T} \operatorname{Tr}_{i}^{-T} L_{i} .
$$

With this notation, and by elimination of $\stackrel{\circ}{U}_{i}$, the original problem $(22)$ reduces to the form $[16,23]$

$$
S \bar{u}=F \quad \text { in } \quad \mathrm{V} .
$$

3.2. The abstract Neumann-Neumann preconditioner. For the abstract problem given by (25), it seems natural to precondition the sum $S=\sum \bar{R}_{i}^{T} S_{i} \bar{R}_{i}$ by a weighted sum of the inverses $M^{-1}=\sum D_{i} S_{i}^{-1} D_{i}^{T}$, as proposed among other in $[1,7,32]$.

We propose a two level generalisation of this algorithm in order to handle the multidomain case and corner singularities. This framework is a generalization of the earlier Neumann-Neumann preconditioners with a coarse space [27]. Suppose that $S$ and $S_{i}$ are positive self-adjoint operators, and that $S$ is coercive on V. Our method is defined by the following choices:

1. a partition of unity $D_{i}: \mathrm{V}_{i} \rightarrow \mathrm{V}$ satisfying

$$
\sum_{i=1}^{N} D_{i} \bar{R}_{i}=\left.I\right|_{\mathrm{V}} ;
$$

2. a local coarse space $\bar{Z}_{i}$ containing potential local singularities, such that

$$
\operatorname{ker} S_{i} \subset \bar{Z}_{i} \subset \mathrm{V}_{i} \text {; }
$$

3. a space $V_{i}^{o}$ that contains a complement of $\bar{Z}_{i}$

$$
\mathrm{V}_{i}=\mathrm{V}_{i}^{o}+\bar{Z}_{i},
$$

on which $S_{i}$ is coercive.

We then introduce

1. the global coarse space

$$
\mathrm{V}_{o}=\sum_{i=1}^{N} D_{i} \bar{Z}_{i},
$$

2. the $S$ orthogonal projection $P: \mathrm{V} \rightarrow \mathrm{V}_{o}$, 
3. the inverse $S_{i o}^{-1}: \mathrm{V}_{i}^{o} \rightarrow \mathrm{V}_{i}^{o}$ defined by the solution $S_{i o}^{-1} L$ of the local variational problem

$$
S_{i o}^{-1} L \in V_{i}^{o}: \quad\left\langle S_{i}\left(S_{i o}^{-1} L\right), v\right\rangle=\langle L, v\rangle, \quad \forall v \in V_{i}^{o},
$$

4. the local fine space

$$
\mathrm{V}_{i}^{\perp}=(I-P) D_{i} \mathrm{~V}_{i}^{o} \subset \mathrm{V}
$$

equipped with the scalar product

$$
b_{i}\left(\bar{u}_{i}, \bar{v}_{i}\right)=\left\langle S_{i} \bar{u}_{i}^{o}, \bar{v}_{i}^{o}\right\rangle,
$$

where $\bar{u}_{i}^{o}, \bar{v}_{i}^{o}$ are uniquely defined by

$$
\bar{u}_{i}^{o}, \bar{v}_{i}^{o} \in \mathrm{V}_{i}^{o o}, \quad(I-P) D_{i} \bar{u}_{i}^{o}=\bar{u}_{i}, \quad(I-P) D_{i} \bar{v}_{i}^{o}=\bar{v}_{i},
$$

with

$$
\mathrm{V}_{i}^{o o}=\left\{\bar{v}_{i} \in \mathrm{V}_{i}^{o}:\left\langle S_{i} \bar{v}_{i}, \bar{z}_{i}\right\rangle=0, \forall \bar{z}_{i} \in \mathrm{V}_{i}^{o} \cap \operatorname{ker}(I-P) D_{i}\right\} .
$$

The proposed preconditioner is then an abstract additive Schwartz algorithm $[38,16]$ operating on the bilinear form

$$
b(\bar{u}, \bar{v})=\langle S \bar{u}, \bar{v}\rangle
$$

and using the decomposition

$$
\mathrm{V}=\mathrm{V}_{o}+\sum_{i} \mathrm{~V}_{i}^{\perp}
$$

with the scalar products $b_{i}(\cdot, \cdot)$ on the space $\mathrm{V}_{i}^{\perp}$ and $b(\cdot, \cdot)$ on $\mathrm{V}_{o}$. That is, the preconditioner is defined by

$$
M^{-1}: V \rightarrow V, \quad M^{-1}: \bar{r} \mapsto \bar{u}=\bar{u}_{o}+\sum_{i} \bar{u}_{i},
$$

where $\bar{u}_{o}, \bar{u}_{i}$ are solutions of the variational problems

$$
\begin{array}{lll}
\bar{u}_{o} \in \mathrm{V}_{o}: & b\left(\bar{u}_{o}, \bar{v}_{o}\right)=\left\langle\bar{r}, \bar{v}_{o}\right\rangle, & \forall \bar{v}_{o} \in \mathrm{V}_{o}, \\
\bar{u}_{i} \in \mathrm{V}_{i}^{\perp}: & b_{i}\left(\bar{u}_{i}, \bar{v}_{i}\right)=\left\langle\bar{r}, \bar{v}_{i}\right\rangle, & \forall \bar{v}_{i} \in \mathrm{V}_{i}^{\perp} .
\end{array}
$$


The input vector $\bar{r}$ to the preconditioner has the meaning of a residual associated with an error vector $\bar{e} \in \mathrm{V}$ by $\bar{r}=S \bar{e}$. From (28) and the definition of $P$, the coarse component is $\bar{u}_{o}=P \bar{e}=S^{-1} \bar{r}$. Substituting

$$
\bar{u}_{i}=(I-P) D_{i} \bar{u}_{i}^{o}, \quad \bar{u}_{i}^{o} \in \mathrm{V}_{i}^{o o},
$$

and using the definition of $b_{i}(\cdot, \cdot)$ shows that $(29)$ is equivalent to finding $\bar{u}_{i}^{o} \in \mathrm{V}_{i}^{o o}$ such that

$$
\left\langle S \bar{u}_{i}^{o}, \bar{v}_{i}^{o}\right\rangle=\left\langle\bar{r},(I-P) D_{i} \bar{v}_{i}^{o}\right\rangle,
$$

for all $\bar{v}_{i}^{o} \in \mathrm{V}_{i}^{o o}$. By definition,

$$
\mathrm{V}_{i}^{o}=\mathrm{V}_{i}^{o o} \oplus\left(V_{i}^{o} \cap \operatorname{ker}(I-P) D_{i}\right), \quad \mathrm{V}_{i}^{o o} \perp_{S_{i}}\left(V_{i}^{o} \cap \operatorname{ker}(I-P) D_{i}\right) .
$$

Let $\bar{v}_{i}^{o} \in V_{i}^{o} \cap \operatorname{ker}(I-P) D_{i}$. Then the right hand side of (31) is zero, and, by (32), the left hand side of (31) is also zero since $\bar{u}_{i}^{o} \in V_{i}^{o o}$. So (31) holds also for all $\bar{v}_{i}^{o} \in V_{i}^{o} \cap \operatorname{ker}(I-P) D_{i}$, and by (32), for all $\bar{v}_{i}^{o} \in V_{i}^{o}$.

We may conclude that

$$
\bar{u}_{i}=(I-P) D_{i} S_{i o}^{-1} D_{i}^{t}(I-P)^{t} \bar{r}=(I-P) D_{i} S_{i o}^{-1} D_{i}^{t} S(I-P) S^{-1} \bar{r}
$$

and obtain the preconditioned operator

$$
M^{-1} S=P+\sum_{i}(I-P) D_{i} S_{i o}^{-1} D_{i}^{t} S(I-P) .
$$

REMARK 3.1. It follows from (33) that in the case when the spaces $V_{i}^{o}$ are chosen so that $V_{i}=V_{i}^{o} \oplus \operatorname{ker} S_{i}$, we recover the abstract Balancing Domain Decomposition algorithm from [27]. This is also clear from the implementaton in Section 3.3 below.

REMARK 3.2. The space $V_{i}^{\perp}$ is independent of the choice of $V_{i}^{o}$, so it is only a function of the coarse space $\bar{Z}_{i}$.

Proof. Take two spaces $\mathrm{V}_{i}^{o}$ and $\mathrm{V}_{i}^{1}$. By assumption, any $\bar{v}_{i}$ in $\mathrm{V}_{i}^{1}$ can be decomposed into

$$
\bar{v}_{i}=\bar{v}_{i}^{o}+\bar{v}_{i}^{z}, \bar{v}_{i}^{o} \in \mathrm{V}_{i}^{o}, \bar{v}_{i}^{z} \in \bar{Z}_{i} .
$$

Hence

$$
\begin{aligned}
(I-P) D_{i} \bar{v}_{i} & =(I-P) D_{i} \bar{v}_{i}^{o}+(I-P) D_{i} \bar{v}_{i}^{z} \\
& =(I-P) D_{i} \bar{v}_{i}^{o} \in(I-P) D_{i} \mathrm{~V}_{i}^{o}
\end{aligned}
$$


Therefore, $V_{i}^{1 \perp}=(I-P) D_{i} \mathrm{~V}_{i}^{1}$ is included in $(I-P) D_{i} \mathrm{~V}_{i}^{o}=V_{i}^{\perp}$. The converse is true by exchanging the roles of $\mathrm{V}_{i}^{o}$ and $\mathrm{V}_{i}^{1}$. $\mathrm{C}$

REMARK 3.3. Although the space $V_{i}^{\perp}$ does not depend on the choice of $V_{i}^{o}$, the scalar product $b_{i}$ and hence the proposed preconditioner does depend on the choice of $V_{i}^{o}$.

3.3. Implementation of the preconditioner. The preconditioner is evaluated within the conjugate gradients method [22] as follows. Given the problem $S \bar{u}=\bar{f}$ and $\bar{u}^{(0)} \in \mathrm{V}$, we first solve the coarse problem

$$
\bar{d}_{o} \in V_{o}: \quad\left\langle S \bar{d}_{o}, \bar{v}_{o}\right\rangle=\left\langle\bar{f}, \bar{v}_{o}\right\rangle-\left\langle S \bar{u}^{(0)}, \bar{v}_{o}\right\rangle, \quad \forall \bar{v}_{o} \in V_{o}
$$

and define the initial approximation for preconditioned conjugate gradients by

$$
\bar{u}^{(1)}=\bar{u}^{(0)}+\bar{d}_{o} .
$$

Now the initial error, related to the initial residual by $\bar{r}^{(1)}=\bar{f}-S \bar{u}^{(1)}=S \bar{e}^{(1)}$, satisfies $\left\langle S e^{(1)}, \bar{v}_{o}\right\rangle=0$ for all $\bar{v}_{o} \in V_{o}$. Thus $P \bar{e}^{(1)}=0$. So, from (33), the preconditioned residual in the first step of conjugate gradients is

$$
(34) I^{-1} \bar{r}^{(k)}=P \bar{e}^{(1)}+\sum_{i}(I-P) D_{i} S_{i o}^{-1} D_{i}^{t} S(I-P) \bar{e}^{(k)}=\sum_{i}(I-P) D_{i} S_{i o}^{-1} D_{i}^{t} \bar{r}^{(k)}
$$

with $k=1$. Since in each step $k$ of conjugate gradients, the approximate solution is a linear combination of the initial approximation $\bar{u}^{(1)}$ and previous preconditioned residuals, we have by induction $P \bar{e}^{(k)}=0$, and hence (34) holds for all $k$. To evaluate (34), we solve then the $N$ independent subdomain problems (these local problems are well posed since $S_{i}$ is coercive on $\mathrm{V}_{i}^{o}$ by construction)

$$
\bar{u}_{i} \in \mathrm{V}_{i}^{o}: \quad\left\langle S_{i} \bar{u}_{i}, \bar{v}_{i}\right\rangle=\left\langle D_{i}^{t} \bar{r}, \bar{v}_{i}\right\rangle, \quad \forall \bar{v}_{i} \in \mathrm{V}_{i}^{o}
$$

followed by the solution of the coarse problem

$$
\bar{u}_{o} \in \mathrm{V}_{o}: \quad\left\langle S \bar{u}_{o}, \bar{v}_{o}\right\rangle=\left\langle\bar{r}, \bar{v}_{o}\right\rangle-\left\langle S \sum_{i} D_{i} \bar{u}_{i}, \bar{v}_{o}\right\rangle, \quad \forall \bar{v}_{o} \in \mathrm{V}_{o}
$$

and put

$$
M^{-1} \bar{r}=\bar{u}_{o}+\sum_{i} D_{i} \bar{u}_{i} .
$$


3.4. Convergence analysis. We have

THEOREM 3.4. The abstract Neumann-Neumann preconditioner given by (33) satisfies

$$
\kappa\left(M^{-1} S\right)=\frac{\lambda_{\max }\left(M^{-1} S\right)}{\lambda_{\min }\left(M^{-1} S\right)} \leq(N+1) \max _{i} \sup _{\bar{v}_{i} \in V_{i}^{o o}} \frac{\left\|D_{i} \bar{v}_{i}^{o}\right\|_{S}^{2}}{\left\|\bar{v}_{i}\right\|_{S_{i}}^{2}},
$$

where $N$ is the maximum number of neighbors of any subdomain, that is,

$$
N=\max _{i}\left|\left\{j: \exists \bar{v}_{i} \in V_{i}, \bar{v}_{j} \in V_{j}:\left\langle S \bar{v}_{i}, \bar{v}_{j}\right\rangle \neq 0\right\}\right| .
$$

Proof. If for some constants $C_{o}, \omega$,

$$
\begin{aligned}
& \forall \bar{v} \in \mathrm{V}, \exists \bar{v}_{o} \in V_{o}, \bar{v}_{i} \in \mathrm{V}_{i}^{\perp}: \quad b_{o}\left(\bar{v}_{o}, \bar{v}_{o}\right)+\sum_{i} b_{i}\left(\bar{v}_{i}, \bar{v}_{i}\right) \leq C_{o}^{2} b(\bar{v}, \bar{v}) \\
& \forall \bar{v}_{i} \in \mathrm{V}_{i}^{\perp}: \quad b_{i}\left(\bar{v}_{i}, \bar{v}_{i}\right) \leq \omega b\left(\bar{v}_{i}, \bar{v}_{i}\right),
\end{aligned}
$$

then the abstract estimate from [16] gives

$$
\kappa\left(M^{-1} S\right) \leq \omega(N+1) C_{o}^{2} .
$$

We first verify the decomposition of unity (35). Let $\bar{v} \in \mathrm{V}$. Then,

$$
\bar{v}=P \bar{v}+(I-P) \bar{v}=\bar{v}_{o}+\bar{v}_{\perp} .
$$

By orthogonality, we have

$$
b\left(\bar{v}_{o}, \bar{v}_{o}\right)+b\left(\bar{v}_{\perp}, \bar{v}_{\perp}\right)=b(\bar{v}, \bar{v}),
$$

and, from the partition of unity (26),

$$
\bar{v}_{\perp}=\sum_{i}(I-P) D_{i} R_{i} \bar{v}_{\perp}=\sum_{i}(I-P) D_{i}\left(R_{i} \bar{v}_{\perp}-\bar{z}_{i}\right):=\sum_{i} \bar{v}_{i}, \quad \bar{v}_{i} \in \mathrm{V}_{i}^{\perp} .
$$

Here, $z_{i} \in \operatorname{ker}(I-P) D_{i}$ is so that $R_{i} \bar{v}_{\perp}-\bar{z}_{i}$ is the $S_{i}$ - orthogonal projection of $R_{i} \bar{v}_{\perp}$ onto $V_{i}^{o o}$ and $(I-P) D_{i}\left(R_{i} \bar{v}_{\perp}-\bar{z}_{i}\right) \in \mathrm{V}_{i}^{\perp}$. From this and the definition of $b_{i}(\cdot, \cdot)$, we get

$$
\begin{aligned}
\sum_{i} b_{i}\left((I-P) D_{i}\left(R_{i} \bar{v}_{\perp}-\bar{z}_{i}\right),(I-P) D_{i}\left(R_{i} \bar{v}_{\perp}-\bar{z}_{i}\right)\right) & =\sum_{i}\left\langle S_{i}\left(R_{i} \bar{v}_{\perp}-\bar{z}_{i}\right), R_{i} \bar{v}_{\perp}-\bar{z}_{i}\right\rangle \\
& \leq \sum_{i}\left\langle S_{i} R_{i} \bar{v}_{\perp}, R_{i} \bar{v}_{\perp}\right\rangle \\
& =\left\langle S \bar{v}_{\perp}, \bar{v}_{\perp}\right\rangle=b\left(\bar{v}_{\perp}, \bar{v}_{\perp}\right) .
\end{aligned}
$$


By this and (37), (35) holds with $C_{o}=1$.

To verify (36), let $\bar{v}_{i} \in \mathrm{V}_{i}^{\perp}$. By definition of $\mathrm{V}_{i}^{o o}, \bar{v}_{i}=(I-P) D_{i} \bar{v}_{i}^{o}$ for some $v_{i}^{o} \in \mathrm{V}_{i}^{o o}$. Using the definition of $b_{i}(\cdot, \cdot)$ and the fact that $P$ is an $S$-orthogonal projection, we get

$$
\begin{aligned}
b_{i}\left(\bar{v}_{i}, \bar{v}_{i}\right) & =\left\langle S_{i} v_{i}^{o}, v_{i}^{o}\right\rangle \\
a\left(\bar{v}_{i}, \bar{v}_{i}\right) & =\left\langle S(I-P) D_{i} v_{i}^{o},(I-P) D_{i} v_{i}^{o}\right\rangle \leq\left\langle S D_{i} v_{i}^{o}, v_{i}^{o}\right\rangle .
\end{aligned}
$$

This gives (36) with

$$
\omega=\max _{i} \sup _{v_{i}^{o} \in \mathrm{V}_{i}^{o o}} \frac{\left\langle S D_{i} v_{i}^{o}, v_{i}^{o}\right\rangle}{\left\langle S_{i} v_{i}^{o}, v_{i}^{o}\right\rangle}
$$

concluding the proof.

3.5. Practical choice of the preconditioner for plate and shells. We now need to choose the injection $D_{i}$ and the local spaces $\bar{Z}_{i}$ and $\mathrm{V}_{i}^{o}$. We suppose here that the finite element space $\mathbb{H}(\Omega)$ is such that each local finite element has the same degrees of freedom as the reduced HCT triangle (one vertical displacement and two rotations per node).

For implementation reasons (flexibility and parallelism), the map $D_{i}$ must be as local as possible. The generic choice consists then in defining $D_{i}$ on each interface degree of by :

$$
D_{i} \bar{v}\left(P_{l}\right)=\frac{\rho_{i}^{s}}{\rho^{s}} \bar{v}\left(P_{k}\right)
$$

if the $l$ degree of freedom of $\mathrm{V}$ corresponds to the $k$ degree of freedom of $\mathrm{V}_{i}$,

$$
D_{i} \bar{v}\left(P_{l}\right)=0,
$$

if not. Here $s \geq 1 / 2, \rho_{i}$ is a local measure of the stiffness of subdomain $\Omega_{i}$ (for example an average Young modulus on $\Omega_{i}$ ) and $\rho^{s}=\sum_{P_{l} \in \Omega_{j}} \rho_{l}^{s}$ is the sum of $\rho^{s}$ on all subdomains $\Omega_{j}$ containing $P_{l}$. In our computations, we choose $s=1$; the choice $s=1 / 2$ was used in [16]. In [28, 29,36], it was realized (though not for plate problems) that any $s \geq 1 / 2$ results in the condition number bounded independently of the values $\rho_{i}$, i.e., independently of coefficient jumps between subdomains.

We will see that the proposed preconditioner is efficient for plate problems if all elements of $\mathrm{V}_{i}^{o o}$ have zero normal displacements $v^{n}\left(P_{c}\right)$ at all corners $P_{c}$ of subdomain $\Omega_{i}$. The first choice of local spaces is then to take

$$
\begin{aligned}
& \mathrm{V}_{i}^{o}=\left\{\bar{v}_{i}=\left.\operatorname{Tr} v_{i}\right|_{\Gamma_{i}} \in \mathrm{V}_{i}: v_{i}^{n}\left(P_{c}\right)=0, \text { for all corners }\left(P_{c}\right) \text { of } \Omega_{i}\right\}, \\
& \bar{Z}_{i}=\left\{\bar{z}_{i} \in \mathrm{V}_{i}:\left\langle S_{i} \bar{z}_{i}, \bar{w}_{i}\right\rangle=0, \forall \bar{w}_{i} \in \mathrm{V}_{i}^{o}\right\} .
\end{aligned}
$$

$\mathrm{RR} \mathrm{n}^{\circ} 2635$ 
The next choice, originally proposed in [25], consists of first decomposing

$$
\mathrm{H}\left(\Omega_{i}\right)=\mathrm{H}^{\circ}\left(\Omega_{i}\right) \oplus P_{1}
$$

and then setting

$$
\left\{\begin{array}{l}
\mathrm{V}_{i}^{o}=\left.\operatorname{Tr} \mathrm{H}^{o}\left(\Omega_{i}\right)\right|_{\Gamma_{i}} \\
\bar{Z}_{i}=\operatorname{Tr} P_{1}+\sum_{\text {corner }} S_{i o}^{-1} L_{c}
\end{array}\right.
$$

with $L_{c}$ a point normal load applied to $P_{c}$. With this choice, we have indeed

$$
v^{n}\left(P_{c}\right)=\left\langle v, L_{c}\right\rangle=\left\langle S_{i} z_{i}^{c}, v\right\rangle \quad \forall v \in \mathrm{V}_{i}^{o} .
$$

Decomposing $z_{i}^{c}$ into $z_{i}^{c}=z_{1}^{c}+z_{o}^{c}$, with $z_{1}^{c} \in \operatorname{Tr} P_{1}$ and $z_{o}^{c} \in \bar{Z}_{i} \cap V_{i}^{o} \subset \operatorname{ker}\left((I-P) D_{i}\right)$, we deduce

$$
v^{n}\left(P_{c}\right)=\left\langle S_{i} z_{1}^{c}, v\right\rangle+\left\langle S_{i} z_{o}^{c}, v\right\rangle=0, \quad \forall v \in \mathrm{V}_{i}^{o o} .
$$

We will analyze both choices of preconditioners.

REMARK 3.5. By definition, a corner is a point of the interface where at least three subdomains meet. We will suppose that each subdomain has at least three nonaligned corners. If not, and if $\partial \Omega_{i} \cap \partial \Omega_{s}$ is empty, we will add a fictitious corner to the corresponding subdomain so that the Schur operator is indeed coercive on $V_{i}^{o}$.

REMARK 3.6. Let $N_{C}$ be the number of corners of subdomain $\Omega_{i}$. The selection (40) results in $\operatorname{dim} Z_{i}=3+N_{C}$, that is, $3+N_{C}$ coarse degrees of freedom for the subdomain, while in (39), we get only $N_{C}$ coarse degrees of freedom. In addition, the columns of $\operatorname{dim} Z_{i}$ in (40) are almost linearly dependent, causing numerical problems, which does not happen in (39).

Also, the solution of the local problem on $V_{i}^{o}$ for (39) is simply solution of the Dirichlet problem with boundary values imposed at corners, while for (40), one has to compute a pseudoinverse solution of a singular problem, which is more troublesome.

\section{Condition Number Estimate for Plate Problems.}

4.1. Technical lemmas. Some of the following lemmas were stated without proofs in [25]. Let us denote by $\Gamma_{i j}$ the interface separating $\Omega_{i}$ from $\Omega_{j}$. Assume that the interface is so smooth that trace theorem and extension theorems hold [37, p. 189]; piecewise $C^{1}$ is sufficient.

The first lemma concerns extension of $H^{1 / 2}$ function by zero and its proof in included only for completeness; cf.[8]. 
Lemma 4.1. If $\left|\frac{\partial u}{\partial s}\right|_{0, \infty, \Gamma_{i j}} \leq \frac{c}{h}|u|_{0, \infty, \Gamma_{i j}}$ and $u=0$ on $\overline{\Gamma_{j}-\Gamma_{i j}}$, then

$$
\left.|u|_{\frac{1}{2}, 2, \Gamma_{j}}^{2} \leq|u|_{\frac{1}{2}, 2, \Gamma_{i j}}^{2}+\left(c^{2}+2 \ln \frac{\left|\Gamma_{i j}\right|}{2 h}\right)|u|_{0, \infty, \Gamma_{i j}}^{2}\right) .
$$

Proof. Let $H_{i j}$ denote the length of $\Gamma_{i j}$. By construction, the $|u|_{\frac{1}{2}, 2, \Gamma_{j}}^{2}$ norm can be written

$$
\begin{aligned}
|u|_{\frac{1}{2}, 2, \Gamma_{j}}^{2}= & |u|_{\frac{1}{2}, 2, \Gamma_{i j}}^{2}+\int_{0}^{H_{i j} / 2} \frac{u^{2}(s)}{s} d s+\int_{H_{i j} / 2}^{H_{i j}} \frac{u^{2}(s)}{H_{i j}-s} d s \\
= & |u|_{\frac{1}{2}, 2, \Gamma_{i j}}^{2}+\int_{0}^{h} \frac{u^{2}(s)+u^{2}\left(H_{i j}-s\right)}{s} d s \\
& +\int_{h}^{H_{i j} / 2} \frac{u^{2}(s)+u^{2}\left(H_{i j}-s\right)}{s} d s .
\end{aligned}
$$

By assumption, since $u(0)=u\left(H_{i j}\right)=0$, we have

$$
\begin{aligned}
|u(s)| & \leq|u|_{0, \infty, \Gamma_{i j}}, \\
|u(s)| & =\left|\int_{0}^{s} \frac{\partial u}{\partial s}(x) d x\right| \leq s\left|\frac{\partial u}{\partial s}\right|_{0, \infty, \Gamma_{i j}}, \\
\left|u\left(H_{i j}-s\right)\right| & =\left|\int_{H_{i j}}^{H_{i j}-s} \frac{\partial u}{\partial s}(x) d x\right| \leq s\left|\frac{\partial u}{\partial s}\right|_{0, \infty, \Gamma_{i j}} .
\end{aligned}
$$

Thus, we get

$$
\begin{aligned}
|u|_{\frac{1}{2}, 2, \Gamma_{j}}^{2} & \leq|u|_{\frac{1}{2}, 2, \Gamma_{i j}}^{2}+2\left|\frac{\partial u}{\partial s}\right|_{0, \infty, \Gamma_{i j}}^{2} \int_{0}^{h} \frac{s^{2}}{s} d s+2|u|_{0, \infty, \Gamma_{i j}}^{2} \int_{h}^{\frac{H_{i j}}{2}} \frac{d s}{s} \\
& \leq|u|_{\frac{1}{2}, 2, \Gamma_{i j}}^{2}+|u|_{0, \infty, \Gamma_{i j}}^{2}\left[c^{2}+2 \ln \frac{H_{i j}}{2 h}\right] .
\end{aligned}
$$

(

The next lemma is an extension of the discrete Sobolev inequality for piecewise linear functions $[8,15]$ to polynomials of arbitrary order, cf., also [33, Theorem 2].

Lemma 4.2. Let $\left(I_{k}\right)_{k}$ be a quasi uniform partition of $(0, H)$ of diameter $h$. Let $V$ consists of all continuous functions whose restriction to $I_{k}$ are polynomials of degree $p$. Then

$$
\sup _{(0, H)}|u|^{2} \leq C(p)\left(1+\ln \frac{H}{h}\right)\left(|u|_{\frac{1}{2}, 2,(0, H)}^{2}+\frac{1}{H}|u|_{0,2,(0, H)}^{2}\right), \forall u \in V .
$$

$\operatorname{RR} \mathrm{n}^{\circ} 2635$ 
Proof. By using a regular map on $(0, H)$, we can reduce ourselves to the case where $I_{k}=[k h,(k+1) h]$. Consider the linear interpolation operator $I: V \rightarrow$ $V_{1}$, which interpolates $v$ by piecewise linear polynomials at points $k \frac{h}{p}$. By finite dimension, we have on each $I_{k}$ and for each $u \in V$

$$
C_{1}(p)|u|_{n, q, I_{k}}^{2} \leq|I u|_{n, q, I_{k}}^{2} \leq C_{2}(p)|u|_{n, q, I_{k}}^{2}, \forall n=0,1, \forall q=2, \infty,
$$

which by summation yields

$$
C_{1}(p)|u|_{n, q,(0, H)}^{2} \leq|I u|_{n, q,(0, H)}^{2} \leq C_{2}(p)|u|_{n, q,(0, H)}^{2}
$$

By interpolation, the above inequalities also hold for $n=\frac{1}{2}$.

On the other hand, using the discrete Sobolev inequality for piecewise linear functions [15], we get

$$
\sup _{(0, H)}|I u|^{2} \leq C(p)\left(1+\ln \frac{H}{h}\right)\left(|I u|_{\frac{1}{2}, 2,(0, H)}^{2}+\frac{1}{H}|I u|_{0,2,(0, H)}^{2}\right) .
$$

The lemma follows then directly by combining (42) and (41).

The next lemma contain the essence of our estimate.

Lemma 4.3. For any $\bar{U}_{i} \in V_{i}$ with zero normal displacement $\bar{U}_{i n}\left(P_{c}\right)$ at corners, we have

$$
\begin{aligned}
& \left|\nabla I_{H C T}\left(D_{i} \bar{U}_{i}\right)\right|_{\frac{1}{2}, 2, \Gamma_{j}}^{2} \\
& \quad \leq C\left(\frac{\rho_{i}^{s}}{\rho_{i}^{s}+\rho_{j}^{s}}\right)^{2}\left(1+\ln \frac{H}{h}\right)^{2}\left[\left|\nabla I_{H C T} \bar{U}_{i}\right|_{\frac{1}{2}, 2, \Gamma_{i}}^{2}+\frac{1}{H}\left|\nabla I_{H C T} \bar{U}_{i}\right|_{0,2, \Gamma_{i}}^{2}\right] .
\end{aligned}
$$

Here, $I_{H C T}$ is the restrition of the HCT interpolation onto the boundary, which is same as Hermite interpolation along the boundary.

Proof. We only treat the case where the boundary $\Gamma_{i} \cap \Gamma_{j}$ is reduced to a segment $\Gamma_{i j}$, the proof to come being easily adaptable to the case $i=j$ or to the case where $\Gamma_{i} \cap \Gamma_{j}$ is reduced to a corner $P_{c}$.

Let $\varphi_{c \alpha}$ denote the nodal shape function of the reduced HCT triangle associated to the degree of freedom $\bar{U}_{\alpha}\left(P_{c}\right)=\partial_{\alpha} \bar{u}\left(P_{C}\right)$. On any regular triangulation, its $H^{2}$ semi-norm $\left|\nabla \varphi_{c \alpha}\right|_{1,2, \Omega}$ is uniformly bounded, and hence by the trace theorem

$$
\left|\nabla \varphi_{c \alpha}\right|_{\frac{1}{2}, 2, \Gamma_{l}} \leq C, \forall \Gamma_{l} \subset \Gamma
$$


Let $I_{i j}$ denote the indicator function of $\Gamma_{i j}$. Then, by construction of $D_{i}$, we have

$$
\begin{aligned}
\left.I_{H C T}\left(D_{i} U_{i}\right)\right|_{\Gamma}= & \frac{\rho_{i}^{s}}{\rho_{i}^{s}+\rho_{j}^{s}} I_{i j}\left[I_{H C T} \bar{U}_{i}-\sum_{\partial \Gamma_{i j}} \sum_{\alpha} \bar{U}_{i \alpha}\left(P_{c}\right) \varphi_{c \alpha}\right] \\
& +\sum_{\partial \Gamma_{i j}} \sum_{\alpha} \frac{\rho_{i}^{s}}{\rho_{i}^{s}+\rho_{j}^{s}+\rho_{l}^{s}+\ldots} \bar{U}_{i \alpha}\left(P_{c}\right) \varphi_{c \alpha} \\
= & \frac{\rho_{i}^{s}}{\rho_{i}^{s}+\rho_{j}^{s}} u_{i c}+\sum_{\partial \Gamma_{i j}} \sum_{\alpha} \rho_{c}^{s} \bar{U}_{i \alpha}\left(P_{c}\right) \varphi_{c \alpha}
\end{aligned}
$$

where $\rho_{c}^{s}=\rho_{i}^{s} /\left(\rho_{i}^{s}+\rho_{j}^{s}+\rho_{l}^{s}+\ldots\right)$, the summation being over all subdomains adjacent to $\Omega_{i}$. By construction, all degrees of freedom of $u_{i c}$ are zero at the corners $\partial \Gamma_{i j}$. Hence $u_{i c}$ and its gradients take zero value on $\partial \Gamma_{i j}$ implying

$$
u_{i c}=\nabla u_{i c}=0 \quad \text { on } \overline{\Gamma_{j}-\Gamma_{i j}} .
$$

Moreover, on each boundary segment $T \cap \Gamma_{i j}$ of the triangulation, $\nabla u_{i c}$ is a second order polynomial, and hence, by a standard finite dimensional argument used on the fixed segment $\frac{1}{h_{T}} T \cap \Gamma_{i j}$, there exists a constant $C$ independent of $h$ such that

$$
\left|\frac{\partial}{\partial s}\left(\nabla u_{i c}\right)\right|_{0, \infty, T \cap \Gamma_{i j}} \leq \frac{C}{h_{T}}\left|\nabla u_{i c}\right|_{0, \infty, T \cap \Gamma_{i j}} .
$$

We can therefore apply Lemma 4.1 to $\nabla u_{i c}$, yielding

$$
\begin{aligned}
\left|\nabla u_{i c}\right|_{\frac{1}{2}, 2, \Gamma_{j}}^{2} & \leq\left(c^{2}+2 \ln \frac{\left|\Gamma_{i j}\right|}{2 h}\right)\left|\nabla u_{i c}\right|_{0, \infty, \Gamma_{i j}}^{2} \\
& +\left|\nabla u_{i c}\right|_{\frac{1}{2}, 2, \Gamma_{i j}}^{2} \\
& \leq C\left(1+\ln \frac{H}{h}\right)\left|\nabla I_{H C T} \bar{U}_{i}\right|_{0, \infty, \Gamma_{i j}}^{2} \\
& +\left|\nabla u_{i c}\right|_{\frac{1}{2}, 2, \Gamma_{i j}}^{2} .
\end{aligned}
$$

By summation, we then get

$$
\begin{gathered}
\left|\nabla I_{H C T}\left(D_{i} \bar{U}_{i}\right)\right|_{\frac{1}{2}, 2, \Gamma_{j}}^{2} \leq 2\left(\frac{\rho_{i}^{s}}{\rho_{i}^{s}+\rho_{j}^{s}}\right)^{2}\left[C\left(1+\ln \frac{H}{h}\right)\left|\nabla I_{H C T} \bar{U}_{i}\right|_{0, \infty, \Gamma_{i j}}^{2}\right. \\
\left.+\left|\nabla u_{i c}\right|_{\frac{1}{2}, 2, \Gamma_{i j}}^{2}\right]+2 \sum_{\partial \Gamma_{i j}} \sum_{\alpha} \rho_{c}^{2 s}\left|\bar{U}_{i \alpha}\left(P_{c}\right)\right|^{2}\left|\nabla \varphi_{c \alpha}\right|_{\frac{1}{2}, 2, \Gamma_{j}}^{2} .
\end{gathered}
$$

RR $\mathrm{n}^{\circ} 2635$ 
Replacing $u_{i c}$ by its value, we can further bound the left hand side by

$$
\begin{aligned}
& \left|\nabla I_{H C T}\left(D_{i} \bar{U}_{i}\right)\right|_{\frac{1}{2}, 2, \Gamma_{j}}^{2} \leq 2\left(\frac{\rho_{i}^{s}}{\rho_{i}^{s}+\rho_{j}^{s}}\right)^{2} C\left(1+\ln \frac{H}{h}\right)\left|\nabla I_{H C T} \bar{U}_{i}\right|_{0, \infty, \Gamma_{i j}}^{2} \\
& \left.\quad+\left.4\left(\frac{\rho_{i}^{s}}{\rho_{i}^{s}+\rho_{j}^{s}}\right)^{2}\left(\mid \nabla I_{H C T} \bar{U}_{i}\right)\right|_{\frac{1}{2}, 2, \Gamma_{i j}} ^{2}+\sum_{\partial \Gamma_{i j}} \sum_{\alpha}\left|\bar{U}_{i \alpha}\left(P_{c}\right)\right|^{2}\left|\nabla \varphi_{c \alpha}\right|_{\frac{1}{2}, 2, \Gamma_{i j}}^{2}\right) \\
& \quad+2 \sum_{\partial \Gamma_{i j}} \sum_{\alpha} \rho_{c}^{2 s}\left|\bar{U}_{i \alpha}\left(P_{c}\right)\right|^{2}\left|\nabla \varphi_{c \alpha}\right|_{\frac{1}{2}, 2, \Gamma_{j}}^{2} \cdot
\end{aligned}
$$

But $\left|\bar{U}_{i \alpha}\left(P_{i}\right)\right|$ is bounded by $\left|\nabla I_{H C T}\left(\bar{U}_{i}\right)\right|_{0, \infty, \Gamma_{i j}}$ and $\left|\nabla \varphi_{c \alpha}\right|_{\frac{1}{2}, 2, \Gamma_{l}}$ is bounded by a constant. Hence, the above inequality implies

$$
\begin{aligned}
& \left|\nabla I_{H C T}\left(D_{i} \bar{U}_{i}\right)\right|_{\frac{1}{2}, 2, \Gamma_{j}}^{2} \\
& \quad \leq 2\left(\frac{\rho_{i}^{s}}{\rho_{i}^{s}+\rho_{j}^{s}}\right)^{2}\left[\left(C^{\prime}+C \ln \frac{H}{h}\right)\left|\nabla I_{H C T}\left(\bar{U}_{i}\right)\right|_{0, \infty, \Gamma_{i j}}^{2}+2\left|\nabla I_{H C T}\left(\bar{U}_{i}\right)\right|_{\frac{1}{2}, 2, \Gamma_{i j}}^{2}\right] .
\end{aligned}
$$

The result now follows by a direct application of Lemma 4.2 .

In the preconditioning step, neighboring subdomains exchange information through their common boundary and then extend these averaged traces by discrete $a_{i}$-harmonic functions inside each subdomain. The following theorem guarantees that this extension is bounded even for plate-like operators.

THEOREM 4.4. Consider a shape regular locally uniform grid made of triangles or quadrilaterals, on a given domain $\Omega$. Partition each quadrilateral into two triangles and let $\mathbb{H}_{h}$ be the HCT finite element space constructed on this triangulation. Let $\left.v \in \operatorname{Tr} \mathbb{H}_{h}\right|_{\partial \Omega}$ be given. Then, there exists $\left.\operatorname{Ext}(v) \in \mathbb{H}_{h}\right|_{\Omega}$ such that

$$
\begin{gathered}
\left.\operatorname{Tr} \operatorname{Ext}(v)\right|_{\partial \Omega}=v \\
|\operatorname{Ext}(v)|_{2,2, \Omega} \leq C|\nabla v|_{\frac{1}{2}, 2, \partial \Omega} .
\end{gathered}
$$

Proof. Let $H_{h}^{2}(\Omega)$ be the space

$$
H_{h}^{2}=\left\{v \in H^{2}(\Omega),\left.\left.\operatorname{Tr} v\right|_{\partial \Omega} \in \operatorname{Tr} \mathbb{H}_{h}\right|_{\partial \Omega}\right\} .
$$

Let $\tilde{v} \in H_{h}^{2}$ be the solution of the non homogeneous Dirichlet continuous plate problem

$$
\begin{aligned}
& a(\tilde{v}, w)=0, \forall w \in H_{0}^{2}(\Omega), \\
& \left.\operatorname{Tr} \tilde{v}\right|_{\partial \Omega}=v .
\end{aligned}
$$


We then construct $\operatorname{Ext}(v) \in \mathbb{H}_{h}$ by averaging $\tilde{v}$ around each node of the triangulation as follows.

Let $c<1$ be a fixed number and $h_{l}$ be the discretization step around the node $a_{l}$. Let $B_{l}=B\left(a_{l}, c h_{l}\right)$ be the ball centered in $a_{l}$ and of radius $c h_{l}$. We suppose that $c$ is such that $\frac{3}{2} B_{l}$ is included in $\Omega$ for each internal node $a_{l}$. For each internal node $a_{l}$ and for $|\alpha| \leq 1$, we compute

$$
\partial_{\alpha} \bar{v}_{l}=\frac{1}{\left|B_{l}\right|} \int_{B_{l}} \partial_{\alpha} \tilde{v} d x
$$

For each boundary node, we simply set

$$
\partial_{\alpha} \bar{v}_{l}=\partial_{\alpha} \tilde{v}\left(P_{l}\right)=\partial_{\alpha} v\left(P_{l}\right)
$$

From these nodal values $\partial_{\alpha} \bar{v}_{l}$, we finally construct $\operatorname{Ext}(v)$ by

$$
\operatorname{Ext}(v)=\sum_{l \in \bar{\Omega}} \partial_{\alpha} \bar{v}_{l} \varphi_{l \alpha}(x)
$$

with $\varphi_{l \alpha}$ denoting the nodal shape function associated to the degree of freedom $\bar{V}_{l \alpha}=\partial_{\alpha} \bar{v}\left(a_{l}\right)$.

This construction is quite classical and can be found for example in Widlund [16]. By construction, $\operatorname{Ext}(v)$ satisfies (43). The verification of (44) requires two steps.

Step 1 : Bounding $|\operatorname{Ext}(v)|$ locally.

Let $T$ be a given finite element of $\Omega$. Let $a_{i} a_{j}$ an edge of $T$, with vertices $a_{i}$ and $a_{j}$. From Theorem 2.1, we have

$$
|\operatorname{Ext} v|_{2,2, T}^{2} \leq C \sum_{i} Q_{i i+1}(\operatorname{Ext} v) .
$$

To bound $Q_{i j}(E x t v)$, on any internal edge $a_{i} a_{j}$, we construct a box $B_{i j}$ of length $\left|a_{i} a_{j}\right|(1+3 c)$ and width $3 c\left|a_{i} a_{j}\right|$ (Fig. 4). If the triangulation is regular and locally uniform, $B_{i j}$ is included in $\Omega, B_{i}$ and $B_{j}$ are included in $B_{i j}$ and the radii $c h_{i}$ and $c h_{j}$ of $B_{i}$ and $B_{j}$ are greater than $\frac{c}{2}\left|a_{i} a_{j}\right|$.

Let then dilate $B_{i j}$ by the factor $\frac{1}{\left|a_{i} a_{j}\right|}$. The image of $B_{i j}$ is then a fixed ball $\hat{B}$. After dilatation, $\left|\hat{B}_{i}\right|$ and $\left|\hat{B}_{j}\right|$ are bounded below and we have

$$
\left|a_{i} a_{j}\right|^{|\alpha|}\left|\partial_{\alpha} \bar{v}_{i}\right|=\frac{1}{\left|\hat{B}_{i}\right|}\left|\int_{\hat{B}_{i}} \frac{\partial \hat{\tilde{v}}}{\partial \hat{x}_{\alpha}} d \hat{x}\right| \leq\left|\hat{B}_{i}\right|^{-\frac{1}{2}}\left|\frac{\partial \hat{\tilde{v}}}{\partial \hat{x}_{\alpha}}\right|_{0,2, \hat{B}_{i}} \leq C\|\hat{\tilde{v}}\|_{2,2, \hat{B}} .
$$




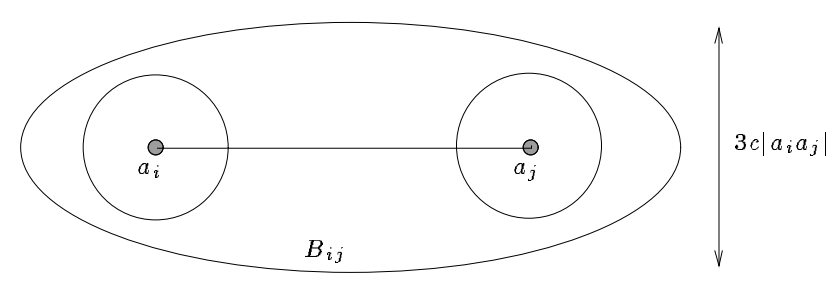

FIG. 4. Representation of the local balls $B_{i}$ and $B_{j}$, and construction of the box $B_{i j}$ for any internal edge.

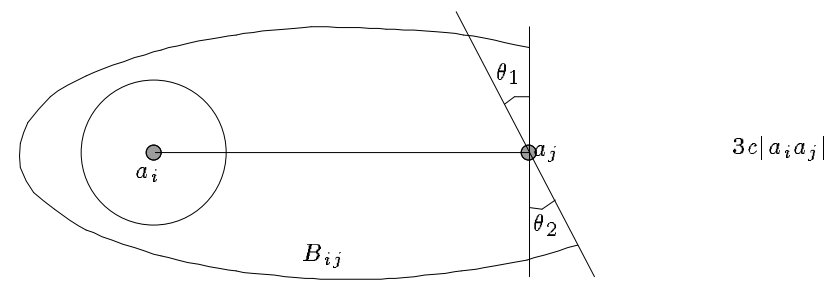

FIG. 5. Representation of $B_{i}$ and construction of the box $B_{i j}$ for any edge intersecting the boundary.

Hence, by summation, we have by construction of $Q_{i j}$

$$
\left|a_{i} a_{j}\right|^{2} Q_{i j}(\bar{v}) \leq C\|\hat{\tilde{v}}\|_{2,2, \hat{B}}^{2}
$$

But $Q_{i j}(\bar{v})$ stays unchanged if we add to $\tilde{v}$ any linear function $\varphi \in P_{1}\left(B_{i j}\right)$, that is if we add to $\hat{\tilde{v}}$ any linear function $\hat{\varphi} \in P_{1}(\hat{B})$. We therefore have

$$
\left|a_{i} a_{j}\right|^{2} Q_{i j}(\bar{v}) \leq C \inf _{\hat{\varphi} \in P_{1}(\hat{B})}\|\hat{\tilde{v}}-\hat{\varphi}\|_{2,2, \hat{B}}^{2} \leq C|\hat{\tilde{v}}|_{2,2, \hat{B}}^{2} \leq C\left|a_{i} a_{j}\right|^{2}|\tilde{v}|_{2,2, B_{i j}}^{2} .
$$

If the edge $a_{i} a_{j}$ has a vertex $a_{j}$ on the boundary, the above construction must be slightly modified. First the box $B_{i j}$ will then be the truncated box shown on Figure 5, with $\theta_{1}$ and $\theta_{2}$ bounded away from $+\frac{\Pi}{2}$ and $-\frac{\Pi}{2}$. Next, from the discrete equivalence of norms on the space of piecewise cubic polynomials on $\partial \hat{\Omega} \cap B\left(\hat{a}_{j}, \frac{c}{2}\right)$ and from the trace theorem, we still have after dilatation

$$
\left|a_{i} a_{j}\right|^{2|\alpha|}\left|\partial_{\alpha} \bar{v}_{j}\right|^{2} \leq\|\hat{v}\|_{\frac{3}{2}, 2, \partial \hat{\Omega} \cap B\left(\hat{a}_{j}, \frac{c}{2}\right)}^{2} \leq C\|\hat{\tilde{v}}\|_{2,2, \hat{B}_{i j}}^{2}
$$

From (51), we then have as before

$$
\left|a_{i} a_{j}\right|^{2} Q_{i j}(\bar{v}) \leq C\|\hat{\tilde{v}}-\hat{\varphi}\|_{2,2, \hat{B}_{i j}}^{2} \leq C|\hat{\tilde{v}}|_{2,2, \hat{B}_{i j}}^{2} \leq C\left|a_{i} a_{j}\right|^{2}|\tilde{v}|_{2,2, B_{i j}}^{2} .
$$


As for any boundary edge, we first observe that

$$
\left|a_{i} a_{j}\right|^{2} Q_{i j}(\bar{v}) \text { and }\left|a_{i} a_{j}\right|^{2}|\nabla v|_{\frac{1}{2}, 2, a_{i} a_{j}}^{2}
$$

are invariant under dilatation. But after dilatation, these two quadratic forms operate on the same finite dimensional space $P_{3}(0,1)$ and the kernel of $\left|a_{i} a_{j}\right|^{2}|\nabla v|_{\frac{1}{2}, 2, a_{i} a_{j}}^{2}$ is included in the kernel of $Q_{i j}$. Hence, there exists again $C$ independent of $a_{i} a_{j}$ such that

$$
\left|a_{i} a_{j}\right|^{2} Q_{i j}(\bar{v}) \leq C\left|a_{i} a_{j}\right|^{2}|\nabla v|_{\frac{1}{2}, 2, a_{i} a_{j}}^{2} .
$$

Step 2. Final result.

From Theorem 2.1, we first have

$$
\begin{aligned}
|\operatorname{Ext}(v)|_{2,2, \Omega}^{2} & =\sum_{T}|\operatorname{Ext}(v)|_{2,2, T}^{2} \\
& \leq C_{0} \sum_{T} \sum_{i \in T} Q_{i i+1}(\bar{v}) \\
& \leq 2 C_{0} \sum_{i i+1 \subset \Omega} Q_{i i+1}(\bar{v})+C_{0} \sum_{i i+1 \subset \partial \Omega} Q_{i i+1}(v) .
\end{aligned}
$$

From Step 1, this implies

$$
|\operatorname{Ext}(v)|_{2,2, \Omega}^{2} \leq 2 C_{0} C \sum_{i i+1 \subset \Omega}|\tilde{v}|_{2,2, B_{i i+1}}^{2}+C_{0} C \sum_{i i+1 \subset \partial \Omega}\left|\frac{\partial v}{\partial x}\right|_{\frac{1}{2}, 2, a_{i} a_{i+1}}^{2} .
$$

In the above sum on all vertices $i$, any domain $B_{i j}$ is covered at most $n_{v}$ times, with $n_{v}$ the maximum number of edges sharing one node. Therefore, we get

$$
|\operatorname{Ext}(v)|_{2,2, \Omega}^{2} \leq 2 n_{v} C_{0} C|\tilde{v}|_{2,2, \Omega}^{2}+C_{0} C|\nabla v|_{\frac{1}{2}, 2, \partial \Omega}^{2} .
$$

But, by construction, the solution $\tilde{v}$ of (45) satisfies

$$
|\tilde{v}|_{2,2, \Omega} \leq C|\nabla v|_{\frac{1}{2}, 2, \partial \Omega}
$$

which completes the verification of (44). 
4.2. Final convergence theorem for plates. From the general theory the condition number of our improved Neumann-Neumann preconditioned plate interface operator is bounded by the energy norm of the restriction map $D_{i} u_{i}$. An estimate of this norm is the essence of the proof of our final convergence theorem. The principal assumption on the elements is the spectral equivalence (53) below, which we have proved for the DKT, HCT, and non-locking Reissner-Mindlin elements in section 2.3 .

THEOREM 4.5. Let the finite element space $\mathbb{H}(\Omega)$ be such that

1. each local finite element is either triangular or quadrilateral, and it has as degrees of freedom one vertical displacement and two rotations per vertex;

2. the local stiffness matrix $A_{T}$ of each finite element $T \subset \Omega_{j}$ satisfies

$$
\rho_{j} c_{o}\left|I_{H C T} U\right|_{2,2, T}^{2} \leq \frac{1}{t^{3}} U^{t} A_{T} U \leq \rho_{j} c_{1}\left|I_{H C T} U\right|_{2,2, T}^{2}
$$

where $c_{o}$ and $c_{1}$ are independent of the element, of the thickness and of the $\Omega_{i}$ averaged values $\rho_{i}$ of the stiffness coefficients;

3. the triangulation $\{T\}$ and the division of $\Omega$ into $\Omega_{i}$ are shape regular;

4. the partition of unity matrices $D_{i}$ are defined by (38) with $s \geq 1 / 2$;

5. the spaces $V_{o}$ and $\bar{Z}_{i}$ are defined by either (39) or (40).

Then

$$
\operatorname{cond}\left(M^{-1} S\right) \leq C\left(1+\ln \frac{h}{H}\right)^{2}
$$

with the constant $C$ independent of $h, H$, and $\rho_{j}>0$, i.e., independent of coefficient jumps between subdomains.

Proof. We show that the map $D_{i}$ and the space $\mathrm{V}_{i}^{00}$ constructed in Section 3.5 satisfy

$$
\frac{\left\langle S D_{i} \bar{u}_{i}, D_{i} \bar{u}_{i}\right\rangle}{\left\langle S_{i} \bar{u}_{i}, \bar{u}_{i}\right\rangle} \leq C\left(1+\ln \frac{H}{h}\right)^{2}, \quad \forall \bar{u}_{i} \in \mathrm{V}_{i}^{00}
$$

The theorem will then follow from Theorem 3.4.

By summation, the basic assumption (53) yields

$$
\rho_{i} c_{o}\left|I_{H C T} U\right|_{2,2, \Omega_{i}}^{2} \leq \sum_{T \subset \Omega_{i}} \frac{1}{t^{3}} U^{T} A_{T} U \leq \rho_{i} c_{1}\left|I_{H C T} U\right|_{2,2, \Omega_{i}}^{2},
$$

for any vector of degrees of freedom $U$. Let $\bar{U}_{i}$ be the $\Gamma_{i}$ boundary degrees of freedom of $U$ and let $v=I_{H C T} U$ be the function obtained by HCT interpolation on $U$. From 
the $C^{1}$ continuity of the HCT element, $v$ and $\nabla v$ on $\partial \Omega_{i}$ depend on $\bar{U}_{i}$ only. So, we may write $v=I_{H C T} \bar{U}_{i}$ and $\nabla v=\nabla I_{H C T} \bar{U}_{i}$ on $\partial \Omega_{i}$. From the trace theorem, it follows that

$$
\begin{aligned}
& \left|\nabla I_{H C T} U\right|_{\frac{1}{2}, 2, \Gamma_{i}}^{2}+\frac{1}{H}\left|\nabla I_{H C T} \bar{U}\right|_{0,2, \Gamma_{i}}^{2} \\
& \quad=\left|\nabla I_{H C T} \bar{U}_{i}\right|_{\frac{1}{2}, 2, \Gamma_{i}}^{2}+\frac{1}{H}\left|\nabla I_{H C T} \bar{U}_{i}\right|_{0,2, \Gamma_{i}}^{2} \leq c_{2}\left\|I_{H C T} U\right\|_{2,2, \Omega_{i}}^{2},
\end{aligned}
$$

which implies

$$
\left|\nabla I_{H C T} \bar{U}_{i}\right|_{\frac{1}{2}, 2, \Gamma_{i}}^{2}+\frac{1}{H}\left|\nabla I_{H C T} \bar{U}_{i}\right|_{0,2, \Gamma_{i}}^{2} \leq c_{2} \inf _{\left.U\right|_{\partial \Omega_{i}}=\bar{U}_{i}}\left\|I_{H C T} U\right\|_{2,2, \Omega_{i}}^{2} .
$$

Above, the constant $c_{2}$ does depend on the shape (aspect ratio) of the subdomain $\Omega_{i}$, but is independent of its size provided we use the scale invariant norm

$$
\|v\|_{2,2, \Omega_{i}}^{2}=|v|_{2,2, \Omega_{i}}^{2}+\frac{1}{H^{2}}|v|_{1,2, \Omega_{i}}^{2}+\frac{1}{H^{4}}|v|_{0,2, \Omega_{i}}^{2} .
$$

Let us now choose $\bar{u}_{i}$ in $\mathrm{V}_{i}^{00}$. Let $\bar{U}_{i}$ be the corresponding set of interface degrees of freedom. By construction, $I_{H C T} \bar{U}_{i}$ cancels at all corners of $\Omega_{i}$. Hence, since each domain has at least three strictly nonaligned corners, there exists a constant $c_{3}$ independent of the size of the subdomain, but dependent of its shape, such that

$$
\left\|I_{H C T} \bar{U}\right\|_{2,2, \Omega_{i}}^{2} \leq c_{3}\left|I_{H C T} \bar{U}\right|_{2,2, \Omega_{i}}^{2}
$$

Combined with (56), this yields

$$
\left(57\left|\nabla I_{H C T} \bar{U}_{i}\right|_{\frac{1}{2}, 2, \Gamma_{i}}^{2}+\frac{1}{H}\left|\nabla I_{H C T} \bar{U}_{i}\right|_{0,2, \Gamma_{i}}^{2} \leq c_{2} c_{3} \inf _{\left.U\right|_{\partial \Omega_{i}}=\bar{U}_{i}}\left|I_{H C T} U\right|_{2,2, \Omega_{i}}^{2}, \forall \bar{u}_{i} \in \mathrm{V}_{i}^{00} .\right.
$$

On the other hand, from the extension given by Theorem 4.4, we have

$$
\inf _{\left.U\right|_{\partial \Omega_{i}}=\bar{U}_{i}}\left|I_{H C T} U\right|_{2,2, \Omega_{i}}^{2} \leq\left|\operatorname{Ext} I_{H C T} \bar{U}_{i}\right|_{2,2, \Omega_{i}} \leq c_{4}\left|\nabla I_{H C T} \bar{U}_{i}\right|_{\frac{1}{2}, 2, \Gamma_{i}}^{2} .
$$

By taking the infimum over $\left.U\right|_{\partial \Omega_{i}}=\bar{U}_{i}$ in (55), these two inequalities imply

$$
\begin{aligned}
& \frac{c_{o}}{c_{2} c_{3}}\left(\left|\nabla I_{H C T} \bar{U}_{i}\right|_{\frac{1}{2}, 2, \Gamma_{i}}^{2}+\frac{1}{H}\left|\nabla I_{H C T} \bar{U}_{i}\right|_{0,2, \Gamma_{i}}^{2}\right) \\
& (58) \leq \frac{1}{\rho_{i} t^{3}} \inf _{\left.U\right|_{\partial \Omega_{i}}=\bar{U}_{i}} \sum_{T \in \Omega_{i}} U^{T} A_{T} U=\frac{1}{\rho_{i} t^{3}}\left\langle S_{i} \bar{U}_{i}, \bar{U}_{i}\right\rangle \leq c_{1} c_{4}\left|\nabla I_{H C T} \bar{U}_{i}\right|_{\frac{1}{2}, 2, \Gamma_{i}}^{2}, \forall \bar{U}_{i} \in \mathrm{V}_{i}^{00} .
\end{aligned}
$$


Hence, on this local space, the local interface energy norm $\frac{1}{\rho_{i} t^{3}}\left\langle S_{i} \bar{U}_{i}, \bar{U}_{i}\right\rangle$ is equivalent to the interface norm

$$
\left|\nabla I_{H C T} \bar{U}_{i}\right|_{\frac{1}{2}, 2, \Gamma_{i}}^{2}+\frac{1}{H}\left|\nabla I_{H C T} \bar{U}_{i}\right|_{0,2, \Gamma_{i}}^{2} .
$$

Moreover, by construction, $\bar{U}_{i n}$ is zero on all corners of $\Omega_{i}$. Thus, Lemma 4.3 can also be applied. From (58), this implies

$$
\begin{aligned}
& \frac{1}{t^{3}}\left\langle S_{j} D_{i} \bar{U}_{i}, D_{i} \bar{U}_{i}\right\rangle \leq \rho_{j} c_{1} c_{4}\left|\nabla I_{H C T}\left(D_{i} \bar{U}_{i}\right)\right|_{\frac{1}{2}, 2, \Gamma_{j}}^{2} \\
& \quad \leq \rho_{j} C c_{1} c_{4}\left(1+\ln \frac{H}{h}\right)^{2}\left[\left|\nabla I_{H C T} \bar{U}_{i}\right|_{\frac{1}{2}, 2, \Gamma_{i}}^{2}+\frac{1}{H}\left|\nabla I_{H C T} \bar{U}_{i}\right|_{0,2, \Gamma_{i}}^{2}\right]\left(\frac{\rho_{i}^{s}}{\rho_{i}^{s}+\rho_{j}^{s}}\right)^{2} \\
& \quad \leq C \frac{c_{1} c_{2} c_{3} c_{4}}{c_{0}}\left(1+\ln \frac{H}{h}\right)^{2} \frac{1}{t^{3}}\left\langle S_{i} \bar{U}_{i}, \bar{U}_{i}\right\rangle
\end{aligned}
$$

since $\rho_{j}\left(\frac{\rho_{i}^{s}}{\rho_{i}^{s}+\rho_{j}^{s}}\right)^{2} \leq C(s) \rho_{i}$ (dividing by $\rho_{i}$ and using compactess of the unit sphere), which is the desired estimates (54). $\square$

Observe also that the same result holds for any elliptic choice of the elasticity tensor $K$, that is for any isotropic or anisotropic Kirchhoff-Love plate model.

\section{Numerical Results.}

5.1. Plate Problems. We validate our proposed solution strategy by numerical tests of increasing complexity.

The purpose of the first test was to confirm the theory and demonstrate the effect of adding corner functions on the condition numbers.

In all tables, neum is the standard Neumann-Neumann preconditionner $\left(\bar{Z}_{i}=\right.$ $\{0\})$, coarse is the general balancing preconditionner with coarse grid solver $\left(\bar{Z}_{i}=P_{1}\right)$, corner-f is the preconditionner using the second choice spaces (40), which is the method of [25], and corner-d uses the first choice of spaces (39).

We show on the following tables the number iter of conjugate gradient iterations, the condition number cond of $M^{-1} S$, the set up CPU time and the CPU time for running the conjugate gradient iterations.

All experiments show that adding the corner functions improves the condition number considerably. The condition numbers were estimated from Ritz values in the Krylov space generated by conjugate gradients. The stopping criterion imposes that the ratio of the $\ell^{2}$ norm of the residual and the right hand side be less than $\varepsilon=10^{-6}$. In all experiments, the domain and the subdomains remain the same, and the elements are uniformly refined. 


\begin{tabular}{|l|l|r|r|r|r|r|}
\hline & ndsd & $\mathrm{h}$ & iter & cond & setup & iter \\
\hline DKT element & 16 & $\mathrm{~h}$ & 90 & $.1 \mathrm{E}+10$ & 4.6 & 3.6 \\
Neumann & 64 & $\mathrm{~h}$ & 305 & $.11 \mathrm{E}+10$ & 22.9 & 16.7 \\
& 16 & $\mathrm{~h} / 2$ & 99 & $.18 \mathrm{E}+11$ & 13.7 & 13.9 \\
& 64 & $\mathrm{~h} / 2$ & 365 & $.2 \mathrm{E}+11$ & 26.6 & 56.7 \\
& 16 & $\mathrm{~h} / 4$ & 108 & $.33 \mathrm{E}+12$ & 60.8 & 61.9 \\
& 64 & $\mathrm{~h} / 4$ & 422 & $.39 \mathrm{E}+12$ & 64.5 & 236.5 \\
\hline DKT element & 16 & $\mathrm{~h}$ & 13 & 3.9 & 5.13 & 0.88 \\
coarse & 64 & $\mathrm{~h}$ & 19 & 15.5 & 25.3 & 3.6 \\
& 16 & $\mathrm{~h} / 2$ & 15 & 5.18 & 15.28 & 2.59 \\
& 64 & $\mathrm{~h} / 2$ & 24 & 23 & 31.1 & 6.5 \\
& 16 & $\mathrm{~h} / 4$ & 16 & 6.5 & 66.94 & 10.3 \\
& 64 & $\mathrm{~h} / 4$ & 29 & 30.9 & 75.6 & 19.6 \\
\hline DKT element & 16 & $\mathrm{~h}$ & 12 & 3.6 & 5.36 & 0.85 \\
corner-f & 64 & $\mathrm{~h}$ & 13 & 4.1 & 27.4 & 3.48 \\
& 16 & $\mathrm{~h} / 2$ & 14 & 4.57 & 16.09 & 2.5 \\
& 64 & $\mathrm{~h} / 2$ & 17 & 6.3 & 35.2 & \\
& 16 & $\mathrm{~h} / 4$ & 15 & 5.6 & 70.2 & 9.75 \\
& 64 & $\mathrm{~h} / 4$ & 21 & 9.19 & 86.5 & 15.26 \\
\hline
\end{tabular}

Results for rectangular plate 


\begin{tabular}{|l|l|r|r|r|r|}
\hline & $\mathrm{h}$ & iter & cond & setup & iter \\
\hline HCT element & $\mathrm{h}$ & 43 & 153 & 14.8 & 3.6 \\
coarse & $\mathrm{h} / 2$ & 59 & 588 & 25.8 & 7.6 \\
& $\mathrm{~h} / 4$ & 75 & 981 & 53.9 & 27.7 \\
\hline HCT element & $\mathrm{h}$ & 16 & 7.8 & 15.8 & 2.9 \\
corner-f & $\mathrm{h} / 2$ & 23 & 22.2 & 26.0 & 4.3 \\
& $\mathrm{~h} / 4$ & 33 & 76.0 & 57.8 & 13.2 \\
\hline DKT element & $\mathrm{h}$ & 33 & 62 & 14.9 & 3.2 \\
coarse & $\mathrm{h} / 2$ & 49 & 239 & 25.1 & 6.5 \\
& $\mathrm{~h} / 4$ & 65 & 898 & 51.3 & 23.8 \\
\hline DKT element & $\mathrm{h}$ & 12 & 3.3 & 15.4 & 2.6 \\
corner-f & $\mathrm{h} / 2$ & 17 & 7.4 & 25.7 & 3.8 \\
& $\mathrm{~h} / 4$ & 25 & 25.1 & 56.6 & 10.8 \\
\hline DKT element & $\mathrm{h}$ & 13 & 3.3 & 4.86 & $(\mathrm{C} 90)$ \\
corner-d & $\mathrm{h} / 2$ & 18 & 7.4 & 8.74 & (C90) \\
& $\mathrm{h} / 4$ & 25 & 27 & 19.24 & (C90) \\
\hline \multicolumn{5}{|c|}{ Results for oval plate with 24 subdomains }
\end{tabular}

The first test considers a clamped rectangular plate (Tab. 1), meshed uniformly and partitioned into rectangular regular subdomains. The results here confirm perfectly the theory. The introduction of corner modes improves the overall condition number and removes virtually all sensitivity to $h$. The number of iterations is not strongly affected either by the number of subdomains.

To determine if adding the corner functions results in an improvement for a realistic problem, we considered next an oval plate (Fig. 2) discretized by an irregular mesh decomposed in 24 subdomains (Tab. 2). Because of the irregularity of the subdomain interface, this case is not completely covered by the theory.

Nevertheless, the tests demonstrate the efficiency of the proposed updated preconditioner and show that the improvement in the CPU times for the iterations outweigh the slight increase in the setup time due to the larger dimension of the coarse space. The plate was clamped on the whole boundary. The CPU for the modified choice of corner space is a total CPU time obtained on a Cray C90 and cannot be compared directly to the other CPU times. Nevertheless, on the C90, the CPU were identical for both choices of corner spaces (4.93 against 4.86, 8.90 against 8.74, 19.80 against 19.24). 


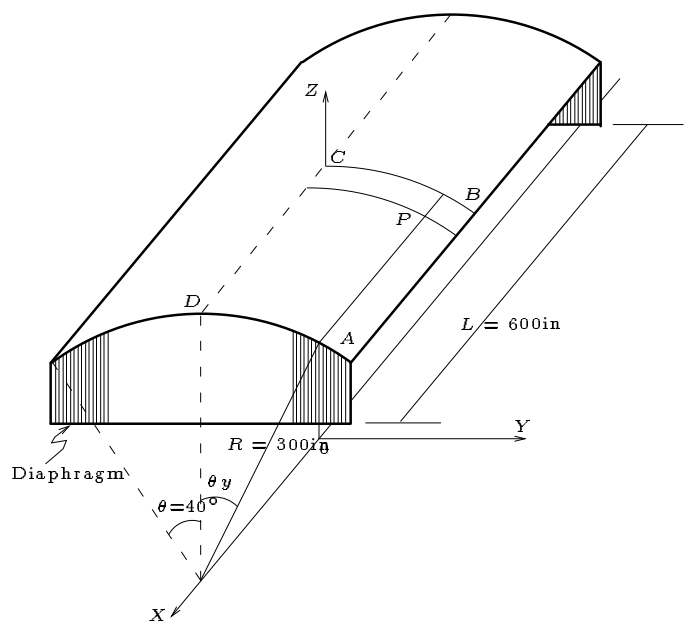

FIG. 6. Description of the shell

5.2. The cylindrical roof. We next turn to the numerical solution of shell problems, using either planar linear DKT finite elements with a specific treatment of the sixth degree of freedom (element $S H E 3$ of University of Colorado at Boulder [3]) or curved geometrically exact nonlinear DKT elements (= DKT) [24].

The aim of the first shell example is to illustrate the behavior of the different preconditioning techniques when dealing with shallow shell problems.

The problem is a standard test case and considers a cylindrical roof, which is supported at its two ends and has its longitudinal edges free. The shell is subjected to a simple gravitational loading (figure 6 ). For symmetry reasons only a quarter of the roof is considered. Three embedded finite element meshes are considered (they are called $\mathrm{h}, \mathrm{h} / 2, \mathrm{~h} / 4)$. The first one has 1152 triangular elements with 625 nodes (3750 degrees of freedom).

The next meshes are obtained from the first one by global regular refinement and have 4608 elements (2401 nodes and 14406 d.o.f) and 18432 elements (9409 nodes and 56454 d.o.f), respectively. All three meshes were decomposed into 24 subdomains as shown in figure 7 , the same decomposition being used in each case. The different results are summarized in Table 3 . These tests were run on one processor of a C98 Cray computer at the I.D.R.I.S. Computer Center in Paris, using the SHE3 finite element. 


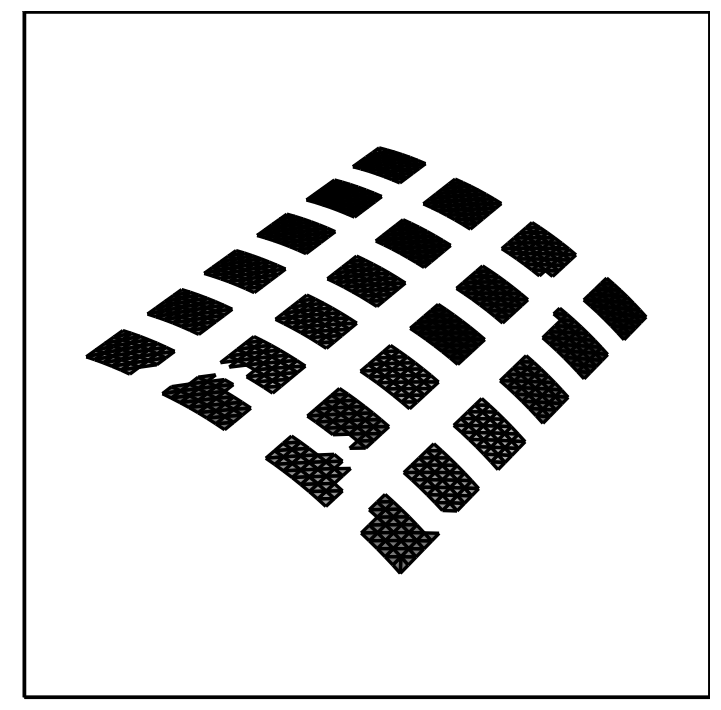

FIG. 7. Mesh decomposition

\begin{tabular}{|c||c|c|c|c|}
\hline & precond & \# iter & condition number & CPU \\
& & & & \\
\hline $\mathrm{h}$ & neum & 201 & $0.36924 \mathrm{E}+08$ & $6.35+9.24=15.59$ \\
$\mathrm{~h} / 2$ & neum & 216 & $0.42081 \mathrm{E}+14$ & $13.42+31.84=45.26$ \\
$\mathrm{~h} / 4$ & neum & 258 & $0.48505 \mathrm{E}+15$ & $54.70+145.43=200.1$ \\
\hline $\mathrm{h}$ & coarse & 41 & $0.10875 \mathrm{E}+03$ & $7.41+2.48=9.89$ \\
$\mathrm{~h} / 2$ & coarse & 50 & $0.27429 \mathrm{E}+03$ & $16.29+8.04=24.33$ \\
$\mathrm{~h} / 4$ & coarse & 63 & $0.66634 \mathrm{E}+03$ & $64.59+36.87=101.39$ \\
\hline $\mathrm{h}$ & corner-f & 24 & $0.26951 \mathrm{E}+02$ & $8.17+1.77=9.94$ \\
$\mathrm{~h} / 2$ & corner-f & 31 & $0.39217 \mathrm{E}+02$ & $18.33+5.37=23.7$ \\
$\mathrm{~h} / 4$ & corner-f & 38 & $0.76593 \mathrm{E}+02$ & $72.15+22.66=94.80$ \\
\hline
\end{tabular}

Convergence of the roof test problem. 
The last test (finer mesh and corner-f preconditionner) was also run on a HP735 workstation. The times were $607.58 \mathrm{~s}$ for the preparation step and $600.54 \mathrm{~s}$ for the solution step (to be compared with $72.15 \mathrm{~s}$ and $22.68 \mathrm{~s}$ on the C98).

We next compare on the coarse mesh the performances of the different choices of coarse spaces for different values of thickness and different choices of finite elements (Table 4). As expected, the results are sensitive to the presence of corner elements in $Z_{i}$, but not to the particular choice of these corner elements. For such an irregular partition, the presence of corner modes improves significantly the condition number of the proposed preconditioner, but here the preconditioner remains sensitive to the mesh refinements and to the plate thickness.

\begin{tabular}{|c|c|c|c|c|c|}
\hline Coarse & & & & & \\
\hline $\mathrm{t}$ & nbiter & $\lambda_{\min }$ & $\lambda_{\max }$ & condit & element \\
\hline 3. & 29 & $.10103 \mathrm{E}+01$ & $.28524 \mathrm{E}+02$ & $.28232 \mathrm{E}+02$ & SHE3 \\
\hline 3. & 37 & $.10114 \mathrm{E}+01$ & $.59672 \mathrm{E}+02$ & $.58999 \mathrm{E}+02$ & DKT \\
\hline 0.3 & 41 & $.10102 \mathrm{E}+01$ & $.10986 \mathrm{E}+03$ & $.10875 \mathrm{E}+03$ & SHE3 \\
\hline 0.3 & 47 & $.10120 \mathrm{E}+01$ & $.13111 \mathrm{E}+03$ & $.12957 \mathrm{E}+03$ & DKT \\
\hline $\begin{array}{c}\text { Corner-f } \\
t\end{array}$ & nbiter & $\lambda_{\min }$ & $\lambda_{\max }$ & condit & element \\
\hline 3. & 18 & $.10117 \mathrm{E}+01$ & $.79302 \mathrm{E}+01$ & $.78384 \mathrm{E}+01$ & SHE3 \\
\hline 3. & 34 & $.10122 \mathrm{E}+01$ & $.38161 \mathrm{E}+02$ & $.37700 \mathrm{E}+02$ & DKT \\
\hline 0.3 & 24 & $.10186 \mathrm{E}+01$ & $.27507 \mathrm{E}+02$ & $.27006 \mathrm{E}+02$ & SHE3 \\
\hline 0.3 & 34 & $.10140 \mathrm{E}+01$ & $.82800 \mathrm{E}+02$ & $.81657 \mathrm{E}+02$ & DKT \\
\hline 0.03 & 64 & $.10176 \mathrm{E}+01$ & $.57399 \mathrm{E}+03$ & $.56404 \mathrm{E}+03$ & SHE3 \\
\hline 0.03 & 57 & $.10100 \mathrm{E}+01$ & $.45691 \mathrm{E}+03$ & $.45238 \mathrm{E}+03$ & DKT \\
\hline 0.003 & 270 & $.10075 \mathrm{E}+01$ & $.66162 \mathrm{E}+04$ & $.65672 \mathrm{E}+04$ & SHE3 \\
\hline 0.003 & 140 & $.10199 \mathrm{E}+01$ & $.10670 \mathrm{E}+04$ & $.10462 \mathrm{E}+04$ & DKT \\
\hline $\begin{array}{c}\text { Corner-d } \\
t\end{array}$ & nbiter & $\lambda_{\min }$ & $\lambda_{\max }$ & condit & element \\
\hline 3. & 18 & $.10208 \mathrm{E}+01$ & $.12032 \mathrm{E}+02$ & $.11787 \mathrm{E}+02$ & SHE3 \\
\hline 3. & 32 & $.10147 \mathrm{E}+01$ & $.34690 \mathrm{E}+02$ & $.34189 \mathrm{E}+02$ & $\mathrm{DKT}$ \\
\hline 0.3 & failure & & & & SHE3 \\
\hline 0.3 & 33 & $.10158 \mathrm{E}+01$ & $.59912 \mathrm{E}+02$ & $.58978 \mathrm{E}+02$ & DKT \\
\hline
\end{tabular}

Observe also that the nonlinear DKT element is slighty more robust, but yield larger condition numbers. Actually, the penalisation of the sixth degree of freedom 


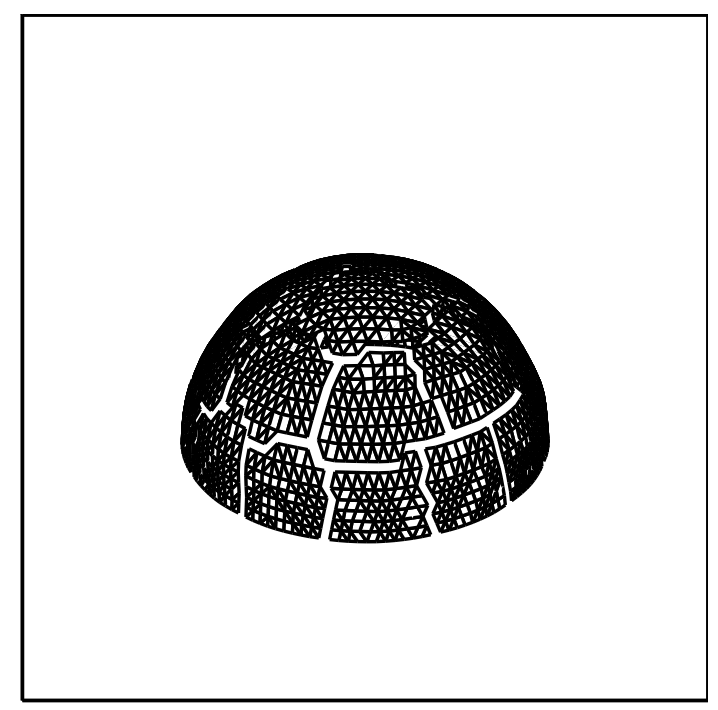

FIG. 8. Deap half sphere

in the planar DKT element leads to algebraic problems as soon as two corners are present (and hence fixed) within the same finite element. Finally, as expected, the performances deteriorate when the thickness decreases because of the resulting singular behavior of the membrane stiffness.

5.3. Deep Half Sphere. The next test problem considers a deep spherical shell of radius 5 subjected to a gravitational loading (Figure 8). The original mesh $h$ contains 2650 planar DKT elements, and is partitioned into 24 subdomains.

Each element is then cut into 4 ( mesh $h / 2)$, and 16 ( mesh $h / 4)$. We compare again in this test case the performances of the different choices of coarse spaces for different thickness and different mesh refinements. The results (Tab. 5) are quite similar to what is observed for the above shallow cylindrical roof.

The corresponding size of the coarse problem for the coarse mesh is coarse 72, corner-f 174, corner-d 306. All three displacements are blocked at corners in the corner-d case, which explains its larger dimension.

5.4. A curved piped under internal pressure. The aim of this test is to show the ability of the method to handle large real-life shell problems.

The simulation computes the deformations of a curved aortic blood vessel subjected to an internal blood pressure as computed by a CFD code. The vessel wall 


\begin{tabular}{|c|c|c|c|c|c|}
\hline \multicolumn{6}{|l|}{ Coarse } \\
\hline $\mathrm{t}$ & nbiter & $\lambda_{\min }$ & $\lambda_{\max }$ & condit & mesh \\
\hline \multirow[t]{3}{*}{.3} & 55 & $0.10141 \mathrm{E}+01$ & $0.34626 \mathrm{E}+03$ & $0.34145 \mathrm{E}+03$ & $\mathrm{~h}$ \\
\hline & 73 & $0.10124 \mathrm{E}+01$ & $0.12476 \mathrm{E}+04$ & $0.12323 \mathrm{E}+04$ & $\mathrm{~h} / 2$ \\
\hline & 95 & $0.10111 \mathrm{E}+01$ & $0.52231 \mathrm{E}+04$ & $0.51657 \mathrm{E}+04$ & $\mathrm{~h} / 4$ \\
\hline \multirow[t]{3}{*}{.03} & 92 & $0.10118 \mathrm{E}+01$ & $0.78075 \mathrm{E}+03$ & $0.77161 \mathrm{E}+03$ & $\mathrm{~h}$ \\
\hline & 106 & $0.10123 \mathrm{E}+01$ & $0.11416 \mathrm{E}+04$ & $0.11277 \mathrm{E}+04$ & $\mathrm{~h} / 2$ \\
\hline & 128 & $0.10112 \mathrm{E}+01$ & $0.23326 \mathrm{E}+04$ & $0.23068 \mathrm{E}+04$ & $\mathrm{~h} / 4$ \\
\hline \multicolumn{6}{|l|}{ Corner-f } \\
\hline $\mathrm{t}$ & nbiter & $\lambda_{\min }$ & $\lambda_{\max }$ & condit & mesh \\
\hline \multirow[t]{3}{*}{.3} & 26 & $0.10146 \mathrm{E}+01$ & $0.18167 \mathrm{E}+02$ & $0.17906 \mathrm{E}+02$ & $\mathrm{~h}$ \\
\hline & 36 & $0.10119 \mathrm{E}+01$ & $0.51838 \mathrm{E}+02$ & $0.51230 \mathrm{E}+02$ & $\mathrm{~h} / 2$ \\
\hline & 53 & $0.10102 \mathrm{E}+01$ & $0.16376 \mathrm{E}+03$ & $0.16211 \mathrm{E}+03$ & $\mathrm{~h} / 4$ \\
\hline \multirow[t]{3}{*}{.03} & 48 & $0.10117 \mathrm{E}+01$ & $0.75847 \mathrm{E}+02$ & $0.74974 \mathrm{E}+02$ & $\mathrm{~h}$ \\
\hline & 59 & $0.10118 \mathrm{E}+01$ & $0.11888 \mathrm{E}+03$ & $0.11749 \mathrm{E}+03$ & $\mathrm{~h} / 2$ \\
\hline & 73 & $0.10111 \mathrm{E}+01$ & $0.20446 \mathrm{E}+03$ & $0.20221 \mathrm{E}+03$ & $\mathrm{~h} / 4$ \\
\hline \multicolumn{6}{|l|}{ Corner-d } \\
\hline \multirow[t]{3}{*}{.3} & 18 & $0.10172 \mathrm{E}+01$ & $0.92599 \mathrm{E}+01$ & $0.91031 \mathrm{E}+01$ & $\mathrm{~h}$ \\
\hline & 27 & $0.10144 \mathrm{E}+01$ & $0.26452 \mathrm{E}+02$ & $0.26077 \mathrm{E}+02$ & $\mathrm{~h} / 2$ \\
\hline & 40 & $0.10153 \mathrm{E}+01$ & $0.62246 \mathrm{E}+02$ & $0.61306 \mathrm{E}+02$ & $\mathrm{~h} / 4$ \\
\hline \multirow[t]{3}{*}{.03} & 24 & $0.10169 \mathrm{E}+01$ & $0.18162 \mathrm{E}+02$ & $0.17859 \mathrm{E}+02$ & $\mathrm{~h}$ \\
\hline & 28 & $0.10177 \mathrm{E}+01$ & $0.38631 \mathrm{E}+02$ & $0.37958 \mathrm{E}+02$ & $\mathrm{~h} / 2$ \\
\hline & 37 & $0.10156 \mathrm{E}+01$ & $0.86683 \mathrm{E}+02$ & $0.85347 \mathrm{E}+02$ & $\mathrm{~h} / 4$ \\
\hline
\end{tabular}

Condition Number for the spherical shell 


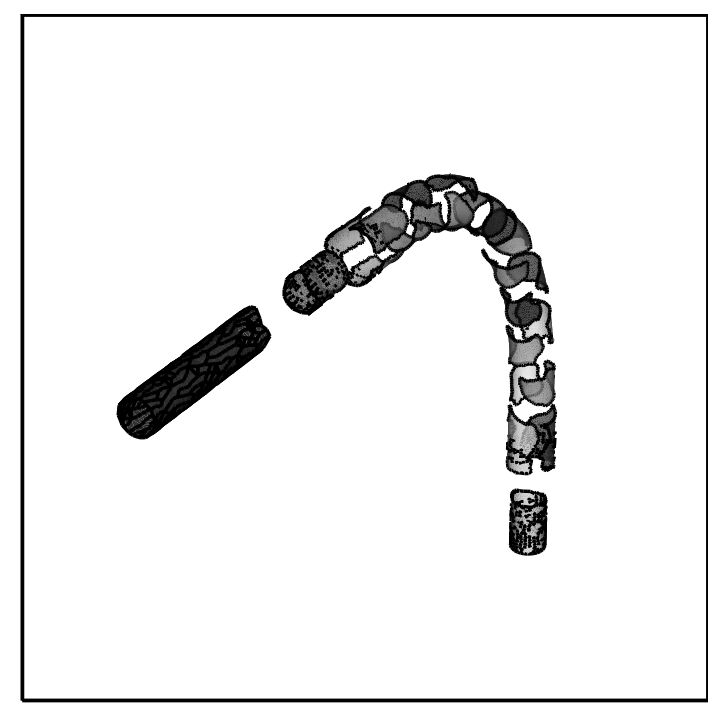

FIG. 9. Mesh of the curved pipe

was 0.125 thick (one tenth of the pipe radius), its Young modulus was $E=3 * 10^{7}$, its Poisson ratio of $\nu=.3$.

The mesh contains 19707 SHE3 elements and 10814 nodes. This mesh was automatically partitioned into 24 subdomains (Fig. 9). The deformed configuration is shown in Figure 10. The number of iterations and CPU time for the various preconditionners are reported in table 6 .

\begin{tabular}{|c||c|c|c|}
\hline precond & \# iter & condition number & CPU \\
\hline neum & 336 & $.13866 \mathrm{E}+08$ & $346.33+6984.4=7330.8$ \\
\hline coars & 104 & $.10931 \mathrm{E}+04$ & $699.05+2238.97=2938.02$ \\
\hline coin & 58 & $.14906 \mathrm{E}+03$ & $1085.71+1289.49=2375.2$ \\
\hline
\end{tabular}

Convergence of the biomedical problem.

6. Conclusion. The above theoretical and numerical evidence indicate that we have introduced a domain decomposition strategy which can handle efficiently fourth order plate problems. For regular partitions, the proposed strategy appears 


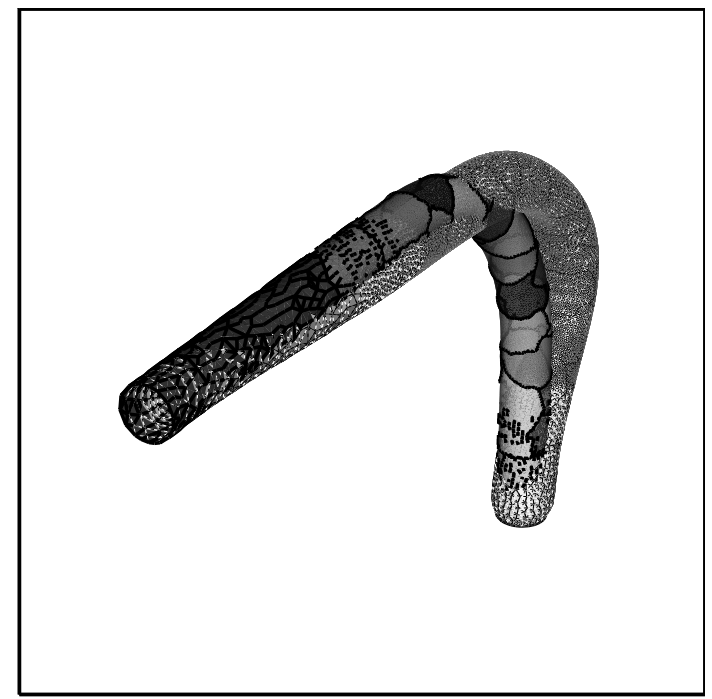

FIG. 10. Curved pipe : deformed configuration

to converge independently of the number of subdomains, even in the case of discontinuous coefficients. On the other hand, the strategy appears to be sensitive to the shape of the subdomains and to the regularity of the interface.

This strategy can also be efficiently extended to quite general shell problems, but the theoretical and numerical results indicate a certain degradation of the results when the shell gets very thin.

\section{REFERENCES}

[1] V. Aghoskov and V. LeBedev, The Poincaré-Steklov's operators and the domain decomposition methods in variational problems, in Computational Processes and Systems, NAUKA, Moscow, 1985, pp. 173-227. In Russian.

[2] J. L. BAtoz, K. J. BATHE, AND W. H. Ho, A study of three-node triangular bending element, Int. J. Numer. Methods Engrg., 15 (1980), pp. 1771-1812.

[3] P. Bergan AND C. FelipPa, A triangular membrane element with rotational degrees of freedom, Comp. Meth. Appl. Mech. Engrg., 50 (1985), pp. 25-69.

[4] M. Bernadou, Méthodes d'éléments finis pour les problèmes de coques minces, Masson, Paris, 1994.

[5] M. Bernadou And J. Boisserie, The Finite Element Method in Thin Shell Theory, Birkhauser, 1982.

[6] A. Blouza And H. Le Dret, Existence et Unicité pour le Modèle de Koiter pour une coque

$\mathrm{RR} \mathrm{n}^{\circ} 2635$ 
peu régulière, C. R. Acad. Sci. Paris Série I, 319 (1994), pp. 1127-1132.

[7] J. F. Bourgat, R. Glowinski, P. LE Tallec, and M. ViDrascu, Variational formulation and algorithm for trace operator in domain decomposition calculations, in Domain Decomposition Methods, T. F. Chan, R. Glowinski, J. Périaux, and O. B. Widlund, eds., SIAM, Philadelphia, 1989, pp. 3-16.

[8] J. H. Bramble, J. E. Pasciak, And A. H. Schatz, The construction of preconditioners for elliptic problems by substructuring, I, Math. Comp., 47 (1986), pp. 103-134.

[9] F. Brezzi And T. Hughes, On drilling degrees of freedom, Computer Methods Appl. Mech. Engrg, 72 (1994), pp. 105-121.

[10] F. Brezzi, T. Hughes, A. Masud, and I. Harari, Finite element with drilling degrees of freedom, in Proc. V Int. Symp. Numer. Meth. Engrg., G. et al, ed., vol. 1, Springer Verlag, 1989, pp. 3-17.

[11] P. G. Ciarlet, Basic error estimates for elliptic problems, in Handbook of Numerical Analysis, P. Ciarlet and J. L. Lions, eds., vol. II, North-Holland, Amsterdam, 1989, pp. 17352 .

[12] L. Cowsar, J. MANDEL, AND M. F. WheELeR, Balancing domain decomposition for mixed finite elements. Math. Comp., to appear in 1995.

[13] Y.-H. De Roeck AND P. Le Tallec, Analysis and test of a local domain decomposition preconditioner, in Fourth International Symposium on Domain Decomposition Methods for Partial Differential Equations, R. Glowinski, Y. Kuznetsov, G. Meurant, J. Périaux, and O. Widlund, eds., SIAM, Philadelphia, PA, 1991.

[14] Y. H. De Roeck, P. Le Tallec, and M. Vidrascu, A domain decomposed solver for nonlinear elasticity, Comp. Meth. Appl. Mech. Engrg, 99 (1992), pp. 187-207.

[15] M. DRYjA, A method of domain decomposition for 3-D finite element problems, in First International Symposium on Domain Decomposition Methods for Partial Differential Equations, R. Glowinski, G. H. Golub, G. A. Meurant, and J. Périaux, eds., Philadelphia, PA, 1988, SIAM.

[16] M. Dryja AND O. B. WidLund, Schwarz methods of Neumann-Neumann type for threedimensional elliptic finite element problems, Comm. Pure Appl. Math, 48 (1995), pp. 121155.

[17] C. FARHAT, Lagrange multiplier based divide and conquer finite element algorithm, J. Comput. Sys. Engrg., 2 (1991), pp. 149-156.

[18] C. FARHAT, J. MANDEL, AND F.-X. Roux, Optimal convergence properties of the FETI domain decomposition method, Comput. Methods Appl. Mech. Engrg., 115 (1994), pp. $367-388$.

[19] C. FARHAT AND F.-X. Roux, Implicit parallel processing in structural mechanics, in Computational Mechanics Advances, J. T. Oden, ed., vol. 2 (1), North-Holland, 1994, pp. 1-124.

[20] C. A. FELIPPA, A survey of parametrized variational principles and application to computational mechanics, Comput. Methods Appl. Mech. Engrg, 113 (1994), pp. 109139.

[21] R. Glowinski And M. F. WheEler, Domain decomposition and mixed finite element methods for elliptic problems, in First International Symposium on Domain Decomposition Methods for Partial Differential Equations, R. Glowinski, G. H. Golub, G. A. Meurant, and J. Périaux, eds., Philadelphia, PA, 1988, SIAM.

[22] G. H. Golub And C. F. Van Loan, Matrix Computations, John Hopkins University Press, 
second ed., 1989.

[23] P. LE TALlec, Domain decomposition methods in computational mechanics, Computational Mechanics Advances, 2 (1994), pp. 121-220.

[24] P. Le Tallec, M. Carrive-Bedouani, and J. Mouro, Finite element approximation of a geometrically exact shell model, Rapport de Recherche 2504, INRIA, Le Chesnay, 1995.

[25] P. Le Tallec, J. Mandel, and M. Vimpascu, Balancing domain decomposition for plates, in Domain Decomposition Methods in Scientific and Engineering Computing, D. E. Keyes and J. Xu, eds., American Mathematical Society, Providence, RI, 1994, pp. 515-524. Proceedings of the 7th International Symposium on Domain Decomposition Methods, Penn State, November 1993.

[26] — Parallel domain decomposition algorithms for solving plate and shell problems, in Advances in Parallel and Vector Processing for Structural Mechanics, Edinburgh, 1994, CIVIL-COMP Ltd. Proceedings, Athens, 1994.

[27] J. MandeL, Balancing domain decomposition, Comm. in Numerical Methods in Engrg., 9 (1993), pp. 233-241.

[28] J. MANDEL AND M. BREzINA, Balancing domain decomposition for problems with large jumps in coefficients. To appear in Mathematics of Computation.

[29] - Balancing domain decomposition: Theory and computations in two and three dimensions, UCD/CCM Report 2, Center for Computational Mathematics, University of Colorado at Denver, 1993.

[30] J. MANDEL AND R. TEZAUR, On the convergence of a substructuring method with Lagrange multipliers, UCD/CCM Report 33, Center for Computational Mathematics, University of Colorado at Denver, December 1994. Submitted.

[31] J. MANDEL, R. TEZAUR, AND C. FARHAT, Optimal lagrange multiplier based domain decomposition method for plate bending problems. Submitted, 1995.

[32] P. MoRICE, Transonic computations by a multidomain technique with potential and Euler solvers, in Symposium Transsonicum III, J. Zienep and H. Oertel, eds., Berlin, 1989, Springer-Verlag.

[33] J. T. Oden, A. PATRA, AND Y. FEng, Parallel domain decomposition solver for adaptive hp finite element methods, TICAM Report 94-11, The University of Texas at Austin, 1994.

[34] J. PitKäRAnta, On a simple finite element method for plate bending problems, in Numerical Techniques in Continuum Mechanics, W. Hackbusch and K. Witsch, eds., no. 16 in Notes on Numerical Fluid Mechanics, Vieweg, Braunschweig/Wiesbaden, 1987, pp. 84101. Proceedings of 2nd GAMM-Seminar, Kiel, January 1996.

[35] F. Roux, Acceleration of the outer conjugate gradient by reorthogonalization for a domain decomposition method with Lagrange multiplier, in Third International Symposium of Domain Decomposition Methods for Partial Differential Equations, T. F. Chan, R. Glowinski, J. Périaux, and O. B. Widlund, eds., Philadelphia, 1990, SIAM, pp. 314-321.

[36] M. SARKIS, Two-level Schwarz methods for nonconforming finite elements and discontinuous coefficients, in Proceedings of the Sixth Copper Mountain Conference on Multigrid Methods, Volume 2, N. D. Melson, T. A. Manteuffel, and S. F. McCormick, eds., no. 3224, Hampton VA, 1993, NASA, pp. 543-566.

[37] E. M. SteIn, Singular Integrals and Defferentiability Properties of Functions, Princeton University Press, 1970.

[38] O. B. WIDLUND, Iterative substructuring methods: Algorithms and theory for elliptic problems in the plane, in First International Symposium on Domain Decomposition Methods for 
Partial Differential Equations, R. Glowinski, G. H. Golub, G. A. Meurant, and J. Périaux, eds., Philadelphia, PA, 1988, SIAM. 
Unité de recherche INRIA Lorraine, Technopôle de Nancy-Brabois 2 Campus scientifique, 615 rue du Jardin Botanique, BP 101, 54600 VILLERS LES NANCY

Unité de recherche INRIA Rennes, Irisa, Campus universitaire de Beaulieu, 35042 RENNES Cedex

Unité de recherche INRIA Rhône-Alpes, 46 avenue Félix Viallet, 38031 GRENOBLE Cedex 1

Unité de recherche INRIA Rocquencourt, Domaine de Voluceau, Rocquencourt, BP 105, 78153 LE CHESNAY Cedex

Unité de recherche INRIA Sophia-Antipolis, 2004 route des Lucioles, BP 93, 06902 SOPHIA-ANTIPOLIS Cedex

Éditeur

INRIA, Domaine de Voluceau, Rocquencourt, BP 105, 78153 LE CHESNAY Cedex (France)

ISSN 0249-6399 